Influence of high mixing intensity on rheology, hydration and microstructure of fresh state cement paste

\title{
Dongyeop Han
}

Engineering Research Institute, Seoul National University, Seoul, South Korea

\section{Raissa Douglas Ferron (corresponding author: rferron@mail.utexas.edu)}

Department of Civil, Architectural, and Environmental Engineering, The University of Texas at Austin, Texas, U.S.

\section{Abstract}

From both the fundamental and applied points of view, there is a growing interest in characterizing the rheological properties of concentrated suspensions, such as cement paste. When a cement paste is sheared, its intrinsic network structure will respond to the shear induced stresses. The influence of mixing intensity on the rheological properties of fresh state cement paste is studied. The results showed that contrary to what is popularly believed, when subjected to a high mixing intensity the rheological properties of cement paste can increase once a certain threshold mixing intensity is achieved. This increase in rheological properties was associated with changes in the chemical nature and physical nature of the cement pastes.

Keywords: mixing, rheology, microstructure, hydration, cement paste

\section{Introduction}

Due to the development of highly flowable concrete and increased use of chemical admixtures, renewed interest in predicting and understanding the rheological behavior of concrete has occurred. In its fresh state, concrete can be considered a suspension consisting of sand and gravel particles suspended in a paste matrix [1,2]; however, even the paste matrix is heterogeneous, comprised of cement particles and hydrates in a pore solution. As a result, the macroscopic flow behavior and rheological properties of 
concrete are highly impacted by the inherent structure of the paste matrix. The factors affecting the rheological properties of pastes can be broadly separated into two categories: non-hydrodynamic factors and hydrodynamic factors. Non-hydrodynamic factors include interactions affects arising from colloidal

32 forces and in the case of cementitious materials, hydration effects. Hydrodynamic effects are due to 33 interactions resulting from non-colloidal origins between the suspending medium and the particles [3-5].

34 Particulate suspensions, such as cement paste, are complex rheological materials whose material 35 properties are dependent upon its processing, especially the mixing process. Understanding the effect of the mixing forces on cement paste is essential to understanding the rheological properties of cement paste [6,7]. Yet the majority of the research on rheological behavior of cement paste has focused on the effect of mixture ingredients and far fewer have focused on the process used to prepare the cement pastes for evaluation. Studying the cement paste phase to gain insight into concrete behavior is common practice; as such it is important to consider how the behavior cement paste is affected by the mixing process. standard uses a high shear mixer for preparation of cement pastes and was motivated from the work of Helmuth et al. [8] who suggested that high mixing intensity ( $\geq 4000 \mathrm{rpm})$ is needed to mimic the mixing energy of cement paste within concrete. Helmuth et al. [8] showed that the correlations between stiffening behavior of concrete prepared in the field and that of cement paste prepared in the lab were dependent on the mixing energy used to prepare the pastes. Good agreement between the stiffening behavior of concrete

47 and pastes mixed using a high shear mixer was seen when the temperature during the high shear mixing process was controlled. However, the shear rate that is experienced by the paste matrix within concrete 49 will depend on the processing stage (e.g. casting, mixing, etc.), as well as the actual mixture composition. 50 For example, Helmuth et al. [8] estimated the shear rate within the paste matrix for a lean concrete 51 mixture during mixing to be $2000 \mathrm{~s}^{-1}$, whereas, the shear rate for a rich concrete paste during mixing was 52 estimated to be $500 \mathrm{~s}^{-1}$. Similarly, Roussel [9] estimated the shear rate applied to cement paste is 53 approximately 5 times higher than the shear rate applied to concrete in the case of ordinary concrete, 
54 whereas it is only approximately 2.5 times higher than the shear rate applied to concrete in the case of 55 SCC. Both works highlight the important role that mixture composition plays on shear rates generated in 56 the pastes and illustrates that as the lubrication layer around the aggregates increases, shear rates in the 57 paste matrix decreases. This implies that a lower mixing speed is needed when mixing cement paste to 58 replicate the mixing that occurs in a rich concrete or SCC than what would needed to replicate the mixing 59 of a lean concrete. Regardless of the composition of the mixture, it is often assumed that as more power 60 is inputted during mixing that a weakening of the internal structure and a reduction in the rheological 61 properties of the mixture will occur. Yield stress is inversely related to slump (slump flow), and thus 62 increasing the mixing power of a cement paste should correspond to an increase in the slump (slump 63 flow) behavior of the paste. However surprisingly the opposite behavior has also been reported in cement 64 paste, mortars and concrete. Rupnow [10] observed a decrease in the temporal evolution of plastic 65 viscosity in pastes prepared with a Hobart and high-shear mixer (maximum mixing speed in both were $66285 \mathrm{rpm})$. However, Helmuth [8] reported higher rates of increase in the yield stress of pastes initially 67 mixed with a high-shear mixer than those initially mixed with Hobart mixer. Similarly, Han and Ferron 68 [11] observed a considerable increase in the rheological properties of cement pastes prepared using a high 69 shear mixer versus those prepared using a Hobart mixer. Further research on the microstructure and rheological properties of fresh state cement paste 71 under high mixing intensity conditions is needed to understand how this affects the fresh state behavior of 72 cement-based materials. The aim of this paper is to examine the influence of high shear mixing on the 73 rheological properties and fresh state microstructure of Portland cement pastes. The fresh state 74 microstructure was assessed with a focused beam reflectance measurement (FBRM) [12-14] device. The 75 results obtained are then discussed on the basis of hydration kinetics and a shear-based agglomeration 76 model, and a mechanism is proposed to explain the effect of high shear mixing on the rheological 77 behavior and microstructural development of the pastes. 


\section{Experimental plan}

80

81 All cement pastes mixtures were prepared using deionized water and a cement conforming ASTM C150

82 Type I and Type II specifications. The chemical composition of the cement is shown in Table 1. Based on

83 the cement mill sheet [15], the Blaine fineness was $401 \mathrm{~m}^{2} / \mathrm{kg}$ and specific gravity was 3.15 . A

84 polycarboxylate-based superplasticizer (herein referred to as "SP") conforming to ASTM C494 [16]

85 Type A and F was employed in many of the mixtures; the specific gravity of the SP was 1.064 [17]. The

86 dosage of SP was set to $0.2 \%$ by mass of the cement; the volume of mixing water was corrected to

87 account for the SP when SP was used. The mixture proportions of the pastes evaluated are provided in

88 Table 2; four different cement pastes with three different solid contents were evaluated. The mixture

89 proportions in Table 2 are expressed for one liter paste; however, for the actual preparation of the cement 90 paste samples, $0.25 \mathrm{~L}$ of cement pastes was prepared.

91

92

93

94

\subsection{Sample preparation}

The mixing protocol for the cement pastes is shown in Figure 1. A Chandler 3060 high shear mixer was used to prepare the pastes (see Figure 2a for a picture of the mixing blade geometry). As shown in Figure 1 , six key steps were conducted to homogenize the pastes. A relatively low mixing speed (500 rpm) was selected for the second step so that differences in the magnitude of the initial mixing intensity and secondary mixing intensity on the rheological behavior of the pastes could be examined. Five different mixing speeds (1000, 2000, 4000, 8000, and $12000 \mathrm{rpm})$ were selected for Steps 4 and 6. A maximum mixing speed of $12000 \mathrm{rpm}$ was selected since this was the maximum mixing speed specified in the 2011 and 2013 versions of ASTM C1738 [18] (note, the ASTM C1738 procedure has been revised and in 2014 standard the maximum speed is $10,000 \mathrm{rpm}$ ). The first four steps should be sufficient to homogenize the pastes, but Step 6 was included to evaluate the influence of additional mixing time on the paste since 
additional mixing is often conducted on high performance cementitious materials [19]. During the mixing

104 process, the mixing speeds were maintained at the pre-set speeds with a control unit. The Chandler high 105 shear mixer was equipped with a water bath. This water bath circulated water around the mixing container 106 and allowed for the temperature of the cement paste to be controlled during mixing. The unit was set to 107 maintain a temperature of $15 \pm 2.0{ }^{\circ} \mathrm{C}$. This was sufficient to maintain limit the temperature variations in 108 pastes prepared at 1000, 2000, and $4000 \mathrm{rpm}$ and the final temperature of these pastes after mixing (i.e., 109 after Step 6) was $24 \pm 2.0^{\circ} \mathrm{C}$. However, even with the water bath system, a $10.0{ }^{\circ} \mathrm{C}$ temperature increase 110 was measured between the temperature of the mixing water before Step 1 and the temperature of the paste 111 after Step 6 (data not shown) for the pastes prepared at 8000 and $12000 \mathrm{rpm}$ during preliminary testing.

112 Therefore, to counteract this temperature increase, in addition to setting the water bath unit to $15 \pm 2.0{ }^{\circ} \mathrm{C}$, 113 the temperature of the mixing water was chilled to $15 \pm 2.0{ }^{\circ} \mathrm{C}$ prior to mixing. With these treatments, the 114 temperature of the pastes mixed at $8000 \mathrm{rpm}$ and $12000 \mathrm{rpm}$ after mixing was approximately $24 \pm 2.0{ }^{\circ} \mathrm{C}$.

\subsection{Experimental methods}

$117 \quad$ 2.3.1. Rheology

118 To evaluate the properties of fresh state cement pastes, flow curve rheological test were conducted using 119 with a commercial rheometer (Anton Paar MCR 301). The rheometer was equipped with a solvent trap to 120 prevent excessive evaporation during the tests and a Peltier setting controlled the temperature of the 121 samples at $23 \pm 1{ }^{\circ} \mathrm{C}$. A concentric cylinder geometry was used; inner diameter of cup and outer 122 dimension of bob were $28.9 \mathrm{~mm}$, and $26.6 \mathrm{~mm}$.

123 Prior to conducting the flow curve test, a $0.1 \mathrm{~s}^{-1}$ preconditioning process was applied for 30 seconds

124 (which was enough time to reach a steady state shear stress condition), followed by 30 seconds of resting.

125 In order to obtain the flow curve, the shear rate was stepped-down from $50 \mathrm{~s}^{-1}$ to $10 \mathrm{~s}^{-1}$ in increments of $12610 \mathrm{~s}^{-1}$ (see Figure 3). Each shear rate was maintained for 60 seconds since this was determined to be a 
sufficient amount of time to achieve a steady-state shear stress for each shear rate. The data was recorded

128 every second and the last 10 data points at a given shear rate were averaged to represent the average

129 resultant shear stress at that shear rate. According to Ferraris et al. [20], the range of typical shear rate

130 experienced by cement paste in concrete during the placing process is 10 or $20 \mathrm{~s}^{-1}$. Since some of the

131 flow curves displayed non-linear behavior, the apparent viscosity at $20 \mathrm{~s}^{-1}$ of shear rate (see equation 1)

132 was used to characterize the mixtures. The apparent viscosity, $\mu_{a p p}$, was defined as:

$$
\mu_{a p p}=\frac{\tau_{20 s^{-1}}}{20 s^{-1}}
$$

where $\tau_{20 s^{-1}}$ is the average resultant shear stress at $20 \mathrm{~s}^{-1}$ of shear rate.

\subsubsection{Fresh State Particle Size Measurements}

137 A focused beam reflectance measurement (FBRM) system was used to evaluate the in-situ particle conditions of the cement pastes $[17,26,31,35]$ and to gain insight about the fresh state microstructure of cement paste. The basic FBRM process involves scanning the suspension at $2 \mathrm{~m} / \mathrm{s}$ with a rotating laser beam and measuring the time duration of the reflected laser from the particles in the scanning path. The time duration of the reflected light coupled with information regarding the scanning velocity of the laser

142 is then used to obtain the chord length of the particles/agglomerates ${ }^{1}$. The chord length of the particle is

143 the length from one edge to the opposite edge on the scanning path rather than the diameter of the 144 particles. Information about the number of chord counts per second, as well as the actual length of the 145 chords, is recorded.

Figure 4 presents a schematic of the FBRM set-up. As can be seen in the figure, the FBRM set-

147 up includes a beaker that contains the sample, the FBRM probe, and an impeller. After preparing the paste

\footnotetext{
${ }^{1}$ In this paper, aggregation and agglomeration will be used interchangeable. Agglomeration is the general process that describes the formation of particles collecting to form a cluster. An agglomerate is a cohesive mass consisting of two or more primary particles.
} 
in the high shear mixer, a portion of the paste was transferred to the FBRM beaker. To minimize the

149 effect of sedimentation on the data, the paste was agitated with the impeller, and the probe was inserted

150 into the sample face to the direction of flow at a downwards angle [12]. Three different agitating speeds

$151(150,300$, and $450 \mathrm{rpm})$ were used to examine how the agitation speed affected fresh state microstructural

152 behavior and agglomeration strength among the particles. Measurements were recorded every 10 seconds,

153 and each test was performed for 30 minutes. Estimating the shear rates of samples in mixing vessels is not

154 trivial, however using a vane approximation (see Equation 2) [24], an indication of the magnitude of the 155 shear rate applied to the sample in the FBRM container at the different mixing speeds was estimated (see 156 Table 3).

$$
\dot{\gamma}=2 R_{b} R_{c} \Omega /\left(R_{c}{ }^{2}-R_{b}{ }^{2}\right)
$$

where $R_{b}$ is the radius of the vane (in this case the radius of the FBRM impeller), $R_{c}$ is the radius of the 159 cup (in this case the radius of the FBRM sample container), and $\Omega$ is the angular velocity of the vane.

160 From Figure 4, it can be seen that $R_{b}=25 \mathrm{~mm}$ and $R_{c}=32.5 \mathrm{~mm}$.

\subsubsection{Calorimetry}

In order to determine the heat signature of the pastes, after preparing the cement pastes with the Chandler high shear mixer, $20 \mathrm{~g}$ of the cement paste was placed in a 3114/3236 TAM Air Thermal, Thermometric AB Isothermal Calorimeter for 20 hours. For each mixture, at least two replicates were tested. The heat of hydration curves in Figure 14, 15, and 16 are based on the results of a representative sample from each

167 mix, while the data in Tables 4, 5, and 6 are based on the average of the samples.

\subsubsection{Flame photometry}

170 Flame photometry was used to gain insight about the pore solution chemistry, particularly the potassium 171 and sodium concentration since they contribute significantly to the ionic strength of the pore solution and 
172 previous work [25] has shown that microstructural rebuilding (i.e. thixotropy) was strongly impacted by 173 these ions [26]. After 045 pastes were prepared (see Section 2.2), a $100 \mathrm{~g}$. sample of fresh state cement 174 paste was put through a Buckner funnel in order to extract the pore solution. A $0.50 \mu \mathrm{m}$ filter paper was 175 use to extract the pore solution. Extraction was conducted by vacuum filtering using the process described 176 by Hema [27]. During the extraction process, $25 \mathrm{ml}$ of deionized water was poured on top of the cement 177 paste and the obtained pore solution was diluted to $0.2 \%(\mathrm{w}: \mathrm{w})$ with deionized water to the detectable 178 range (0 to $199.9 \mathrm{ppm}$ for sodium, and 0 to $9.99 \mathrm{ppm}$ for potassium [28]) of a Cole Parmer Dual Channel 179 Flame Photometer (Model 2655-10).

\section{Results and Discussion}

\subsection{Rheological properties}

183 Figure 5 shows the apparent viscosity values at $20 \mathrm{~s}^{-1}$, and Figure 6 presents the flow curves for the cement paste. The various curves in each sub-plot of Figure 6 correspond to the final mixing speeds that were used to prepare the paste. As expected, the apparent viscosities increased as the solid volume increased. As the solid content increases, particle-to-particle interactions are likely to increase, and the

187 increased interactions among the particles results in an increased resistance to flow [1, 28, 36]. The 188 apparent viscosity values were lower for the 045 SP paste than for the 045 cement paste. This is 189 attributed to the electrosteric hindrance effects from the polycarboxylate-based SP [32,33], and it has been 190 shown that incorporating polycarboxylate--based SP tends to result in weaker agglomerates which can be 191 easily broken down by shear [21,34].

Higher mixing energy has been associated with cement paste with lower viscosities and increased

194 from $1000 \mathrm{rpm}$ to $4000 \mathrm{rpm}$, the apparent viscosity did not increase (it generally decreased or remained 195 the same for all the mixtures). However, when the mixing speed increased above $4000 \mathrm{rpm}$, various 
behaviors were observed. Between 4000-8000 rpm, the apparent viscosity was relatively unchanged for

197 the mixtures without SP; however, a considerable increase in the apparent viscosity values were seen in

198 the pastes containing SP as the sample preparation mixing speed increased from $8000 \mathrm{rpm}$ to $12000 \mathrm{rpm}$.

199 Hence it appears that mixing speeds within a range of $1000 \mathrm{rpm}$ to $4000 \mathrm{rpm}$ is sufficient to disperse the

200 cement particles and that above a threshold mixing speed increasing the speed no longer results in

201 decreasing the viscosity of the cement paste. Cement pastes are often characterized as being Bingham

202 fluids [30], however it can be seen that when the sample preparation mixing speed increased above a

203 certain threshold a shift towards shear-thinning behavior occurred in some of the cement pastes. For the

204 pastes examined in this research, the threshold speed occurred between $4000 \mathrm{rpm}$ and $8000 \mathrm{rpm}$ for the 205 majority of cement pastes (see Figure 6).

\subsection{Microstructural change of cement paste}

208 Figures 7, 8, 9, and 10 display the results of the FBRM experiments and shows how agglomeration 209 evolved over time when samples were prepared using different sample preparation speeds and different 210 FBRM agitation speeds. It can be seen that in all cases, the global behavior of the samples trended 211 towards agglomerate breakup when the agitation speed used during the FBRM experiment increased and 212 as the agitation speed increased generally the chord length of cement pastes decreased. As seen in Figures $2137,8,9$, and 10, at agitation speeds of 150 and $300 \mathrm{rpm}$, the mean chord length of the cement pastes 214 prepared with a final mixing speed of $12000 \mathrm{rpm}$ was generally greater than cement pastes prepared with 215 a final mixing lower than $8000 \mathrm{rpm}$. However, as the FBRM agitation speed increased to $450 \mathrm{rpm}$ (see 216 plot $\mathrm{c}$ in Figures 7, 8, 9, and 10), the chord length of the cement pastes prepared using a final preparation 217 speed of $12000 \mathrm{rpm}$ was often comparable to those prepared with lower sample preparation mixing 218 speeds. The results illustrate the dynamic nature of agglomerate resilience. Samples prepared with the 219 faster sample preparation speeds contained microstructures with larger agglomerates; this may be due to 
stronger interparticle bonds and/or faster rates of agglomeration. Regardless of the mechanism inducing

221 the formation of the agglomerates, it can be seen that these large agglomerates were able to resist

222 breakdown under the lower FBRM agitation speeds of $150 \mathrm{rpm}$ and $300 \mathrm{rpm}$, but when enough power is

223 applied to the system even those agglomerates can be ruptured.

Inspired by an approach described by Jarvis et al. in which the relationship between particle size and velocity gradient is used to determine a shear-based agglomeration strength [38], the slope of the average chord length-FBRM agitation speed curve (see Figure 11) is used as a measure to estimate the 227 overall strength of the microstructure. The curves shown in Figure 11 represent three theoretical examples 228 of a mixture that was prepared using three different sample preparation speeds (A, B, and C) and 229 evaluated using the FBRM technique (note, the mixture composition and the FBRM testing approach is 230 the same in the three examples). For ease of reading, we will refer to the mixture by the sample 231 preparation speed that was used to prepare it (e.g. mixture " $\mathrm{B}$ " is the mixture that is prepared using 232 sample preparation speed B). As the agitation speed increased from "1" to " 2 ", the average chord sizes of 233 all the mixtures decreased. Mixture " $A$ " had the largest initial average particle chord length of the three 234 samples and its final average chord length was the same as mixture "B"; whereas mixture "C" had a slope 235 that is equivalent to mixture " $\mathrm{B}$ ", but the average particle chord length of mixture $\mathrm{C}$ was smaller than that 236 of mixture "B". Since the slope of mixture "B" is the same as mixture "C" the microstructural response of 237 these two mixtures to the agitation is similar, which suggests that the mixtures have equivalent 238 agglomeration strength. However, since the average chord length of mixture "B" is greater than that of 239 mixture " $\mathrm{C}$ ", the microstructure of mixture " $\mathrm{B}$ " is comprised of larger agglomerates than mixture "C"; 240 thus the overall state of agglomeration is considered to be higher than that of mixture "C". Mixture "A" 241 initially has agglomerates that are larger than the other two mixtures, however, as the agitation speed 242 increased, the agglomerate size of mixture "A" decreased to an agglomerate size similar to mixture "B". 243 Thus, the agglomeration strength of mixture " $\mathrm{A}$ " was weaker than mixture " $\mathrm{B}$ ", and this is manifested by 244 the slope of the curve represented by mixture " $\mathrm{A}$ " curve being steeper than that of the curve represented 
by mixture ' $\mathrm{B}$ '. The sign of the slope indicates whether breakdown or agglomeration is occurring, where

246 negative signs represent breakdown/disaggregation has occurred. The absolute magnitude of the slope is

247 used to determine the agglomeration strength, and in the case of a negative slope higher values are

248 associated with lower agglomeration strengths (i.e. weaker systems). Based on this concept, the 249 agglomeration strength of the pastes was calculated by determining the slope of the line passing through 250 the average chord length-FBRM agitation speed plot at $150 \mathrm{rpm}$ and $450 \mathrm{rpm}$.

Figure 12 shows the agglomeration strength of each cement paste as calculated and expressed in

252 Figure 11. It is readily apparent that that the cement paste prepared at higher mixing speeds had weaker 253 agglomeration strength. Furthermore, agglomeration strength decreased as the distance between 254 agglomerates increased, regardless of whether this increased spacing was caused by reducing the number 255 of particles in the system (e.g. compare 042 and 045 mixtures) or via polymeric dispersion (e.g. compare the 045 and 045_SP mixtures). Combining the FBRM chord length results (Figures 7, 8, 9, and 10) and

257 the agglomeration strength results (Figure 12), it is concluded that the cement paste prepared with very 258 high mixing speeds contained larger agglomerates than their counterpart pastes prepared at the lower 259 mixing speeds, however these agglomerates were relatively weak. The results are consistent with work by 260 Ferron [25] that showed that agglomeration strength decreased as agglomerate size increased.

\subsection{Changes in the chemical environment of cement paste}

263 The rheological properties of cement paste are influenced by its microstructure $[6,21,39,40]$, thus when a

264 cement paste is prepared at different mixing speeds, changes are likely to occur to its microstructural state. 265 Agglomerates form not only because of hydration but also due to thixotropy and interparticle forces from 266 the colloidal nature of the finest cement particles [41]. The agglomeration of cement particles can be 267 considered as two different types [26]: coagulation and flocculation. Coagulation refers to a relatively 268 strong and irreversible agglomeration between multiple cement particles (such as those that may occur due to hydration or very strong interparticle forces of attraction), while flocculation is considered as a 
270 relatively weak and reversible agglomeration (such as those that may form due to the thixotropic

271 phenomena). The ionic concentration influences the thickness of electrical double layers of the particles

272 [42]. The Debye-Hückel length (1/א) equation represents the thickness of the double layer (Equation 2)

$273[26,43]:$

274

$$
\frac{1}{\kappa}=\sqrt{\frac{\varepsilon \varepsilon_{0} R T}{2 F^{2} I}}
$$

where $\varepsilon_{0}$ is the permittivity of the vacuum, $\varepsilon$ is the dielectric constant (relative permittivity) of the dispersion medium, $R$ is the gas constant, $T$ is the absolute temperature, $F$ is the Faraday constant, and I

277 is the ionic strength. As the ionic concentration is increased, the thickness of electrical double layers decreases, which can result in increased/stronger agglomeration.

Figure 13 shows an illustration depicting how a high sample preparation mixing intensity may result in increasing agglomeration of cement particles. First, breakdown of cement particles occurs due to the mixing process, thereby increasing the specific surface area in contact with the mixing water. As the specific surface area increases, the ionic concentration of cement paste increases due to an increasing rate of ionic dissolution from the surface of the cement particles [26,44]. This increased ionic concentration 284 reduces the thickness of the diffused layers of the electric double layer on the cement particles, which 285 facilitates agglomeration of cement particles [30, 40, 42]. Thus, it is likely that varying the mixing speed 286 can also result in changing the chemical nature a mixture and that these changes will impact 287 agglomeration kinetics. To investigate this, additional experiments were conducted to examine whether 288 changes in hydration mechanism or concentration of ions in the pore solution occurred when pastes where 289 prepared with different mixing intensities.

The cement pastes were prepared using the protocol shown in Figure 1 and the hydration kinetics of the samples were monitored for 20 hours after initial contact of the cement with the mixing water.

292 Figure 14 shows that as the final sample preparation mixing speed increased, cement hydration was accelerated and the overall heat evolved during this time period increased (see Figure 15); this 
phenomenon occurred regardless of whether the mixture contained superplasticizer or not. Further analysis of the heat of hydration curves (see Tables 4, 5, and 6) elucidates that the end of the acceleration period was more sensitive to the applied mixing speed than the end of the induction period. Hydration 297 kinetics prior to the induction period is controlled by dissolution of ions from the cement grains [46], 298 whereas the acceleration period is controlled by nucleation and growth of $\mathrm{C}-\mathrm{S}-\mathrm{H}$ [47]. Nucleation is a 299 diffusion controlled process. Thus the results suggest that $\mathrm{C}-\mathrm{S}-\mathrm{H}$ formation was modified (e.g. via the rate 300 at which $\mathrm{C}-\mathrm{S}-\mathrm{H}$ is formed and/or the structural arrangement/porosity) since such changes can affect the rate at which ions diffuses through the hydrate. This is in agreement with the results of Juilland et al. [47] who observed a faster rate of hydration in cement pastes that were prepared at $1200 \mathrm{rpm}$ versus those prepared at lower intensities (specifically $500 \mathrm{rpm}$ and hand mixed). It was postulated that this behavior was due an increased dissolution rate of ions from the surface of cement particles caused by the mechanical action of mixing densifying the C-S-H nuclei. Additionally, it cannot be ignored that the formation of the hydration products from the aluminate phases, such as ettringite, will also be occurring during this time period. Similar to the impact of mixing on the silicate phases, it is likely that the increased mixing intensity will also impact the formation of hydrates precipitating from the aluminate phases. Ettringite has a considerable impact on the rheological behavior and stiffening of fresh cement pastes $[40,48]$. As such, changes in ettringite size, morphology, or amount is also likely to have a considerable effect on rheological behavior. Work conducted by Dalas et al. [49] on model system paste 312 mixtures showed that inclusion of polycarboxylate resulted in increasing the specific surface area of 313 ettringite in mixtures containing the polycarboxylate admixture as compared to mixtures not containing 314 polycarboxylate admixtures. Results by $\mathrm{Ng}$ et al. [50] also suggests that increased ettringite formation 315 occurred during the early stages of hydration in pastes containing polycarboxylate admixtures as 316 compared to neat paste. Furthermore, polycarboxylate admixtures are prone to generating air bubbles 317 within the paste matrix and air will also affect rheological properties [51,52]. Thus, the greater sensitivity 318 to mixing intensity seen in the plasticized mixtures as compared to the non-plasticized mixtures could be 
due to the polycarboxylate-based admixture inducing changes within the paste that modified the

320 fundamental rheological response of the paste systems. Generally, an increase in hydration rate is

321 associated with an increase in the early strength development (which is one of the reasons why cement

322 fineness has decreased over the years) [53,54]. However, in many applications, minimizing the initial

323 heat release is preferred in order to limit the development of thermal stresses which can cause early-age

324 cracking. Further work is needed in order to investigate the extent to which mixing intensity influences

325 properties, especially with respect to strength, capillary porosity and shrinkage development.

From Figure 16 (a), it can be seen that for the cement paste prepared at a final mixing speed of $3271000 \mathrm{rpm}$, inclusion of the superplasticizer had a slight retardation effect. Interestingly, the delaying effect of superplasticizers was compensated by increasing the final mixing speed (compare Figures 16 (a), (b), and (c)). Retardation of cement hydration due to superplasticizers has been commonly reported, and could be due to adsorption of the polymer molecules of the superplasticizer onto cement particles inhibiting subsequent topochemical reactions from occurring [50,55-59] and/or an increase in the time needed to reach the percolation threshold due to the particles being initially dispersed further apart from the superplasticizer [53]. It was seen that the apparent viscosity of the 045_SP mixture was lower than of the 045 mixture (see Figure 5) at all the sample preparation mixing speeds that were used in this research. However as the sample preparation speed increased the difference between the apparent viscosity of the 045 and $045 \_S P$ decreased, which indicates that the cement-SP interaction has been affected.

[60]. As depicted in Figure 13, an increase in the ion concentrations of the pore fluid may contribute to the acceleration behavior and particle agglomeration behavior observed in the pastes that were prepared using the higher final mixing speeds. In order to examine whether this could be the case, the concentration of alkali was measured using flame photometry. It can be seen that the potassium and sodium concentrations of the cement paste prepared with final mixing speed of $12000 \mathrm{rpm}$ was higher than other cement pastes prepared with lower final mixing speeds (see Figure 17). Thus the results are in 
344 agreement with the hypothesis that the increased mixing intensity increased the early-age dissolution

345 process. The increased ion concentration in the mixture prepared at $12000 \mathrm{rpm}$ when compared to

346 mixtures prepared at the lower mixing intensities is likely caused by increased deagglomeration of the

347 cement grains due to initial rupturing of the flocs. This deagglomeration could then heighten the

348 accessibility of the alkali ions to the pore solution. Increase soluble alkali content has been shown to

349 increase the rheological properties of pastes [61-62]. This is consistent with the trend seen in Figure 18, in

350 which the mixture containing the highest measured alkali ions displayed the greatest apparent viscosity.

351 Ferron [25] showed that SP demand was strongly correlated to the potassium and sodium concentrations

352 in the pore solution, which suggests that for the paste presented in Figure 17 that mixing at $12000 \mathrm{rpm}$

353 may necessitate more superplasticizer to achieve a target fluidity condition than mixing at $4000 \mathrm{rpm}$.

\section{Conclusion}

356 The results of this study contributes to our understanding of the mechanisms affecting the rheological

357 behavior of cement pastes due to changes in the mixing speed. It was determined that after a certain

358 mixing speed threshold, the rheological properties of fresh state cement paste increased. The results

359 showed that pastes prepared at higher mixing speeds exhibited a larger mean chord size and higher

360 rheological properties than their counterpart pastes prepared at lower mixing speeds. This trend was

361 amplified in pastes containing superplasticizer. Changes in the physical and chemical nature of the pastes

362 occurred when the mixing speed varied. Based on an agglomeration strength analysis using in-situ

363 particle size measurements, pastes that were prepared with high mixing speeds contained larger 364 agglomerates that were more weakly linked together than pastes prepared at lower sample preparation 365 speeds. This behavior was associated with changes in chemical reactions in the cement pastes, specifically 366 with regards to hydration kinetics and soluble alkali concentration. As the mixing speed increases, 
increases in hydration kinetics and alkali content of the pore solution occurred. Such changes will likely lead to a decrease in the thickness of the diffuse double-layer and promote agglomeration.

\section{Acknowledgement}

371 The authors gratefully acknowledge Savitha S. Srinivasan for her assistance in conducting experiments.

372 This material is based upon work supported by the National Science Foundation under Grant No. EEC

373 0927353. Any opinions, findings, and conclusions or recommendations expressed in this material are

374 those of the author(s) and do not necessarily reflect the views of the National Science Foundation.

\section{References}

[1] K. Erdem, K.H. Khayat, A. Yahia, Correlating Rheology of Self-Consolidating Concrete to Corresponding Concrete-Equivalent Mortar, ACI Mater. J. (2010) 154-160.

[2] Z. Toutou, N. Roussel, lop, Multi Scale Experimental Study of Concrete Rheology: From Water Scale to Gravel Scale, Mater. Struct. 39 (2007) 189-199.

[3] C. Viseras, G. Meeten, A. Lopez-Galindo, Pharmaceutical grade phyllosilicate dispersions: the influence of shear history on floc structure, Int. J. Pharm. 182 (1999) 7-20.

[4] P. Coussot, Rheometry of pastes, suspensions, and granular materials: applications in industry and environment, John Wiley \& Sons Inc., New Jersey, 2005.

[5] E. Guazzelli, J. Morris, A physical introduction to suspension dynamics, Cambridge University Press, New York, 2011.

[6] D.A. Williams, A.W. Saak, H.M. Jennings, The influence of mixing on the rheology of fresh cement paste, Cem. Concr. Res. 29 (1999) 1491-1496.

[7] C.F. Ferraris, Measurement of the rheological properties of cement paste: a new approach, in: Int. RILEM Conf. Role Admixtures High Perform. Concr., Monterry, Mexico, 1999: pp. 333-342.

[8] R. Helmuth, L.M. Hills, D.A. Whiting, S. Bhattacharja, Abnormal concrete performance in the presence of admixtures, Portland Cement Association, Skokie, IL, 1995.

[9] N. Roussel, Correlation between Yield Stress and Slump: Comparison between Numerical Simulations and Concrete Rheometers Results, Mater. Struct. 39 (2006) 501-509.

[10] T. Rupnow, V. Schaefer, Investigation of portland cement concrete mix consistency and concrete performance using a two-stage mixing process, Transp. Res. Rec. J. Transp. Res. Board 1979. (2006) 12-20.

398

[11] D. Han, R. Ferron, Effect of mixing method on microstructure and rheology of cement paste, 
Constr. Build. Mater. 93 (2015) 278-288.

[12] J. Whelan, E. Murphy, A. Pearson, Use of focussed beam reflectance measurement (FBRM) for monitoring changes in biomass concentration, Bioprocess Biosyst. Eng. 35 (2012) 963-975.

[13] E. Kougoulos, A. Jones, K. Jennings, M. Wood-Kaczmar, Use of focused beam reflectance measurement (FBRM) and process video imaging (PVI) in a modified mixed suspension mixed product removal (MSMPR) cooling crystallizer, J. Cryst. Growth. 273 (2005) 529-534.

404

[14] P. Barrett, B. Glennon, In-line FBRM Monitoring of Particle Size in Dilute Agitated Suspensions, Part. Part. Syst. Character. 16 (1999) 207-211.

[15] TXI, Type I/II low alkali cement Mill chart, New Braunfels, TX, 2010.

[16] ASTM International, ASTM C494, Standard Specification for Chemical Admixtures for Concrete, ASTM International, West Conshohocken, PA, 2013.

[17] BASF NA Product Regulations, Safety Data Sheet GLENIUM 7700 (MSDS), 2011.

411

412

413

414

415

416

417

[18] ASTM International, ASTM C1738, Standard Practice for High-Shear Mixing of Hydraulic Cement Pastes, ASTM International, West Conshohocken, PA, 2011.

[19] D. Chopin, F. de Larrard, B. Cazacliu, Why do HPC and SCC require a longer mixing time?, Cem. Concr. Res. 34 (2004) 2237-2243.

[20] C.F. Ferraris, J.M. Gaidis, Connection between the rheology of concrete and rheology of cement paste, ACI Mater. J. (1992) 388-393.

[21] R. Ferron, S. Shah, E. Fuente, C. Negro, Aggregation and breakage kinetics of fresh cement paste, Cem. Concr. Res. 50 (2013) 1-10.

[22] A. Blanco, C. Negro, J. Tijero, Flocculation monitoring: focused beam reflectance measurement as a measurement tool, Can. J. 80 (2002) 1-7.

[23] M.-N. Pons, K. Milferstedt, E. Morgenroth, Modeling of chord length distributions, Chem. Eng. Sci. 61 (2006) 3962-3973.

[24] H.A. Barnes, L. Merseyside, J.O. Carnali, The vane-in-cup as a novel rheometer geometry for shear thinning and thixotropic materials, Journal of Rheology. 34 (1990) 841-866.

[25] R.P. Ferron, Formwork Pressure of Self-Consolidating Concrete: Influence of Flocculation Mechanisms, Structural Rebuilding, Thixotropy and Rheology, Ph.D. dissertation, Northwestern University, Evanston, IL, 2008.

[26] M. Yang, C.M. Neubauer, H.M. Jennings, Interparticle Potential and Sedimentation Behavior of Cement Suspensions, Adv. Cem. Based Mater. 5 (1997) 1-7.

[27] J. Hema, The Effects of Liquid Nitrogen on Concrete Hydration, Microstructure, and Properties, Ph.D. dissertation, The University of Texas at Austin, Austin, TX, 2007.

[28] C.-P.I. Company, Model 02655-10 And Model 02655-15 Flame Photometer Operator Manual, Cole-Parmer Instrunment Company, Vernon Hills, IL, 2008.

[29] L.J. Struble, W.G. Lei, Rheological changes associated with setting of cement paste, Adv. Cem. Based Mater. 2 (1995) 224-230.

[30] G. Tattersall, P. Banfill, The rheology of fresh concrete, PITMAN BOOKS LIMITED, London, 1983.

[31] N.A. Tregger, M.E. Pakula, S.P. Shah, Influence of clays on the rheology of cement pastes, Cem. Concr. Res. 40 (2010) 384-391. 
[32] H. Uchikawa, S. Hanehara, D. Sawaki, The role of steric repulsive force in the dispersion of cement particles in fresh paste prepared with organic admixture, Cem. Concr. Res. 27 (1997) 37 50.

[33] C. Li, N. Feng, Y. Li, R. Chen, Effects of polyethlene oxide chains on the performance of polycarboxylate-type water-reducers, Cem. Concr. Res. 35 (2005) 867-873.

[34] J.E. Wallevik, Rheology of Particle Suspensions : Fresh Concrete, Mortar and Cement Paste with Various Types of Lignosulfonates, Norwegian University of Science and Technology, Trondheim, Norway, 2003.

[35] M. Yang, H.M. Jennings, Influences of mixing methods on the microstructure and rheological behavior of cement paste, Adv. Cem. Based Mater. 2 (1995) 70-78.

[36] C.F. Ferraris, K.H. Obla, R. Hill, The influence of mineral admixtures on the rheology of cement paste and concrete, Cem. Concr. Res. 31 (2001) 245-255.

[37] S. Hanehara, K. Yamada, Interaction between cement and chemical admixture from the point of cement hydration, absorption behaviour of admixture, and paste rheology, Cem. Concr. Res. 29 (1999) 1159-1165.

[38] P. Jarvis, B. Jefferson, J. Gregory, S. Parsons, A review of floc strength and breakage, Water Res. 39 (2005) 3121-3137.

[39] S.D. Nair, R.D. Ferron, Set-on-demand concrete, Cem. Concr. Res. 57 (2014) 13-27.

[40] C. Rößler, A. Eberhardt, H. Kučerová, B. Möser, Influence of hydration on the fluidity of normal Portland cement pastes, Cem. Concr. Res. 38 (2008) 897-906.

[41] J.E. Wallevik, Rheological properties of cement paste: Thixotropic behavior and structural breakdown, Cem. Concr. Res. 39 (2009) 14-29.

[42] R. Hunter, Foundations of colloid science, Oxford University Press, New York, 2001.

[43] J.T.G. Overbeek, Interparticle forces in colloid science, Powder Technol. 37 (1984) 195-208.

[44] S.P. Jiang, J.C. Mutin, A. Nonat, Studies on mechanism and physico-chemical parameters at the origin of the cement setting. I. The fundamental processes involved during the cement setting, Cem. Concr. Res. 25 (1995) 779-789.

[45] T. Cosgrove, Colloid science: principles, methods and applications, Ames, Iowa: Blackwell Pub. Professional, 2005.

[46] P. Juilland, E. Gallucci, R. Flatt, K. Scrivener, Dissolution theory applied to the induction period in alite hydration, Cem. Concr. Res. 40 (2010) 831-844.

[47] P. Juilland, A. Kumar, E. Gallucci, R.J. Flatt, K.L. Scrivener, P. Juill, Effect of mixing on the early hydration of alite and OPC systems, Cem. Concr. Res. 42 (2012) 1175-1188.

[48] P.-V. Vlachou, J.-M. Piau, The influence of the shear field on the microstructural and chemical evolution of an oil well cement slurry and its rheometric impact, Cem. Concr. Res. 27 (1997) 869881.

[49] F. Dalas, S. Pourchet, D. Rinaldi, A. Nonat, S. Sabio, M. Mosquet, Modification of the rate of formation and surface area of ettringite by polycarboxylate ether superplasticizers during early C3A-CaSO4 hydration, Cem. Concr. Res. 69 (2015) 105-113.

[50] S. Ng, H. Justnes, Influence of dispersing agents on the rheology and early heat of hydration of blended cements with high loading of calcined marl, Cem. Concr. Compos. 65 (2015) 41-54.

[51] L.Y. Wang, Y.W. Wang, G.B. Gao, Z.P. Chu, Y.S. Liu, K.Y. Wang, et al., Synthesis and 
Performance of Polycarboxylate Superplasticizers with Hydrophobic Side Chains, Appl. Mech. Mater. 357 (2013) 1124-1129.

[52] D. Feys, N. Roussel, R. Verhoeven, G. De Schutter, Influence of air bubbles size and volume fraction on rheological properties of fresh self-compacting concrete, in: 3rd Int. RILEM Symp. Rheol. Cem. Suspens. such as Fresh Concr., RILEM Publications, Reykjavik, Iceland, 2009: pp. 113-120.

[53] D.P. Bentz, E.J. Garboczi, C.J. Haecker, O. Jensen, Effects of cement particle size distribution on performance properties of Portland cement-based materials, Cem. Concr. Res. 29 (1999) $1663-$ 1671.

[54] A.K. Schindler, K.J. Folliard, Heat of hydration models for cementitious materials, ACI Mater. J. 102 (2005) 24-33.

[55] B. Lothenbach, F. Winnefeld, The influence of superplasticizers on the hydration of Portland cement, in: Proceedings of the 12th International Congress on the Chemistry of Cement, Montreal, Canada, 2007: pp. 9-12.

[56] F. Puertas, H. Santos, M. Palacios, S. Martínez-Ramírez, Polycarboxylate superplasticiser admixtures: effect on hydration, microstructure and rheological behaviour in cement pastes, Adv. Cem. Res. 17 (2005) 77-89.

[57] E. Sakai, T. Kasuga, T. Sugiyama, K. Asaga, M. Daimon, Influence of superplasticizers on the hydration of cement and the pore structure of hardened cement, Cem. Concr. Res. 36 (2006) 20492053.

[58] N. Spiratos, M. Pagé, N. Mailvaganam, Superplasticizers for concrete : fundamentals, technology, and practice, Supplementary Cementing Materials for Sustainable Development Inc., Ottawa, 2003.

[59] G. Trtnik, G. Turk, Influence of superplasticizers on the evolution of ultrasonic P-wave velocity through cement pastes at early age, Cem. Concr. Res. 51 (2013) 22-31.

[60] F. Lea, Lea's Chemistry of Cement and Concrete, 4th editio, Elsevier Ltd, Burlington, MA, 2003.

[61] H. Uchikawa, K. Ogawa, S. Uchida, Influence of character of clinker on the early hydration process and rheological property of cement paste, Cem. Concr. Res. 15 (1985) 561-572.

[62] Jiang, S., B.-G. Kim, and P.-C. Aïtcin, Importance of Adequate Soluble Alkali Content to Ensure Cement/Superplasticizer Compatibility, Cem. Concr. Res.. 29(1999): p. 71-78. 


\section{$1 \quad$ List of tables}

2

3 Table 1. Oxide composition of cement [15]

4 Table 2. Mixture proportions for cement paste. c/p refers to solid content volume and is the volume of cement per volume in paste, whereas w/c refers to the water-to-cement ratio and is based on the mass of cement versus the mass of water.

7 Table 3. Estimated shear rate applied in the FBRM set-up

8 Table 4. Influence of mixing speed on the time at which the of induction period ended

9 Table 5. Influence of mixing speed on the time at which the acceleration period ended

10 Table 6. Influence of mixing speed on the slope of acceleration period of cement paste (slope = tangent) 
Table 1. Oxide composition of cement [15]

\begin{tabular}{llllllll}
\hline Items & $\mathrm{SiO}_{2}$ & $\mathrm{Al}_{2} \mathrm{O}_{3}$ & $\mathrm{Fe}_{2} \mathrm{O}_{3}$ & $\mathrm{CaO}$ & $\mathrm{MgO}$ & $\mathrm{SO}_{3}$ & $\mathrm{Na}_{2} \mathrm{O}_{\mathrm{e}}$ \\
\hline Amount (\%) & 20.7 & 4.5 & 3.3 & 65.1 & 1.4 & 2.6 & 0.50 \\
\hline
\end{tabular}

12

13 
Table 2. Mixture proportions for cement paste. $\mathrm{c} / \mathrm{p}$ refers to solid content volume and is the volume of cement per volume in paste, whereas w/c refers to the water-to-cement ratio and is based on the mass of cement versus the mass of water.

\begin{tabular}{|c|c|c|c|c|c|c|}
\hline \multirow{2}{*}{ Code* } & \multirow{2}{*}{$\begin{array}{l}\text { c/p (by } \\
\text { volume) }\end{array}$} & \multirow{2}{*}{$\begin{array}{c}\text { w/c (by } \\
\text { mass) }\end{array}$} & \multirow{2}{*}{ SP } & \multicolumn{3}{|c|}{ Quantity for 1 liter of paste } \\
\hline & & & & Cement $(\mathrm{g})$ & Water $(\mathrm{g})$ & HRWR (g) \\
\hline 042 & 0.42 & 0.44 & \multirow{2}{*}{ None } & 1323.000 & 580.000 & - \\
\hline 045 & & & & 1417.500 & 550.000 & - \\
\hline 045_SP & 0.45 & 0.39 & \multirow{2}{*}{ Yes } & 1417.500 & 547.336 & 2.835 \\
\hline 047_SP & 0.47 & 0.36 & & 1480.500 & 527.217 & 2.961 \\
\hline
\end{tabular}

14 
Table 3. Estimated shear rate applied in the FBRM set-up FBRM agitation speed (rpm) 150 300 Estimated shear rate $\left(\mathrm{s}^{-1}\right)$ 450

118

177

16 
Table 4. Influence of mixing speed on the time at which the of induction period ended

\begin{tabular}{cccccc}
\hline \multirow{2}{*}{ Pastes } & \multicolumn{5}{c}{ Mixing speeds } \\
\cline { 2 - 5 } & $1000 \mathrm{rpm}$ & $2000 \mathrm{rpm}$ & $4000 \mathrm{rpm}$ & $8000 \mathrm{rpm}$ & $12000 \mathrm{rpm}$ \\
\hline O45 & $78 \pm 4 \mathrm{~min}$. & $72 \pm 4 \mathrm{~min}$. & $67 \pm 5 \mathrm{~min}$. & $64 \pm 3 \mathrm{~min}$. & $63 \pm 3 \mathrm{~min}$. \\
045_SP & $87 \pm 3 \mathrm{~min}$. & $83 \pm 3 \mathrm{~min}$. & $78 \pm 5 \mathrm{~min}$. & $74 \pm 2 \mathrm{~min}$. & $69 \pm 5 \mathrm{~min}$. \\
\hline
\end{tabular}

17

18 
Table 5. Influence of mixing speed on the time at which the acceleration period ended

\begin{tabular}{cccccc}
\hline \multirow{2}{*}{ Pastes } & \multicolumn{5}{c}{ Mixing speeds } \\
\cline { 2 - 5 } & $1000 \mathrm{rpm}$ & $2000 \mathrm{rpm}$ & $4000 \mathrm{rpm}$ & $8000 \mathrm{rpm}$ & $12000 \mathrm{rpm}$ \\
\hline O45 & $398 \pm 2 \mathrm{~min}$. & $413 \pm 0 \mathrm{~min}$. & $391 \pm 0 \mathrm{~min}$. & $339 \pm 1 \mathrm{~min}$. & $334 \pm 0 \mathrm{~min}$. \\
045_SP & $455 \pm 1 \mathrm{~min}$. & $455 \pm 0 \mathrm{~min}$. & $428 \pm 0 \mathrm{~min}$. & $377 \pm 7 \mathrm{~min}$. & $331 \pm 0 \mathrm{~min}$. \\
\hline
\end{tabular}

19

20 
Table 6. Influence of mixing speed on the slope of acceleration period of cement paste (slope = tangent)

\begin{tabular}{cccccc}
\hline \multirow{2}{*}{ Pastes } & \multicolumn{5}{c}{ Mixing speeds } \\
\cline { 2 - 6 } & $1000 \mathrm{rpm}$ & $2000 \mathrm{rpm}$ & $4000 \mathrm{rpm}$ & $8000 \mathrm{rpm}$ & $12000 \mathrm{rpm}$ \\
\hline O45 & $2.72 \pm 0.00$ & $2.80 \pm 0.02$ & $2.97 \pm 0.00$ & $3.40 \pm 0.00$ & $3.71 \pm 0.01$ \\
045_SP & $2.57 \pm 0.18$ & $2.81 \pm 0.16$ & $3.27 \pm 0.01$ & $3.95 \pm 0.00$ & $4.40 \pm 0.03$ \\
\hline
\end{tabular}

21 


\section{List of figures}

2 Figure 1. Mixing protocol to prepare cement pastes. Mixing speeds for Steps 4 and 6 ranged from 1000, 2000, 4000, 8000, and $12000 \mathrm{rpm}$.

4 Figure 2. Chandler 3060 mixer system used to prepare cement pastes (a) blade set (b) mixer connected to water circulation system

6 Figure 3. Applied shear rate protocol for flow curve measurement

$7 \quad$ Figure 4. Schematic of FBRM test set up

8 Figure 5. Influence of mixing speed and cement pastes on the apparent viscosity at $20 \mathrm{~s}^{-1}$ of shear rate (the data points were obtained by average of two representative flow curve tests)

Figure 6. Influence of mixing speed on flow curve behaviors depending on different cement pastes

11 Figure 7. Influence of final mixing speeds on particle chord length under different agitation speeds for 042 paste

Figure 8. Influence of final mixing speeds on particle chord length under different agitation speeds for 045 paste

Figure 9. Influence of final mixing speeds on particle chord length under different agitation speeds for 045_SP paste

Figure 10. Influence of final mixing speeds on particle chord length under different agitation speeds for 047_SP paste

Figure 11. Schematic depicting how agglomeration strength of the cement agglomerates can be estimated using average particle chord length and agitation speeds from FBRM experiments

Figure 12. Estimated agglomeration strength in different pastes prepared at different final mixing speeds (Negative values indicates aggregate break-down whereas positive values indicate aggregate build-up.)

24 Figure 13. Schematic view of the hypothesis

25 Figure 14. Influence of the final mixing speeds on rate of heat evolutions of cement pastes

26 Figure 15. Influence of the final mixing speeds on cumulative heat of hydration of cement pastes

27 Figure 16. Influence of mixing speeds on compensating the delaying effect of SP in cement pastes

Figure 17. Influence of applied final mixing speeds on the concentration of alkalis from the fresh state 045 mixture

Figure 18. Relationship between ionic concentrations of alkali ions and apparent viscosity of 045 mixture 
Figure(s)

\begin{tabular}{|c|c|c|c|c|c|}
\hline Step 1. & Step 2. & Step 3. & Step 4. & Step 5. & Step 6. \\
\hline $\begin{array}{c}\text { Water + add } \\
\text { cement }\end{array}$ & $\begin{array}{c}\text { Initial } \\
\text { mixing } \\
\text { (500rpm / } \\
30 \mathrm{~s})\end{array}$ & $\begin{array}{c}\text { Resting } \\
\text { (15s) }\end{array}$ & $\begin{array}{c}\text { Secondary } \\
\text { mixing } \\
\text { (various } \\
\text { speeds /60s) }\end{array}$ & $\begin{array}{c}\text { Resting } \\
\text { (15s) }\end{array}$ & $\begin{array}{c}\text { Additional } \\
\text { mixing } \\
\text { (various } \\
\text { speeds / 120s) }\end{array}$ \\
\hline
\end{tabular}

Figure 1. Mixing protocol to prepare cement pastes. Mixing speeds for Steps 4 and 6 ranged from $1000,2000,4000,8000$, and $12000 \mathrm{rpm}$. 


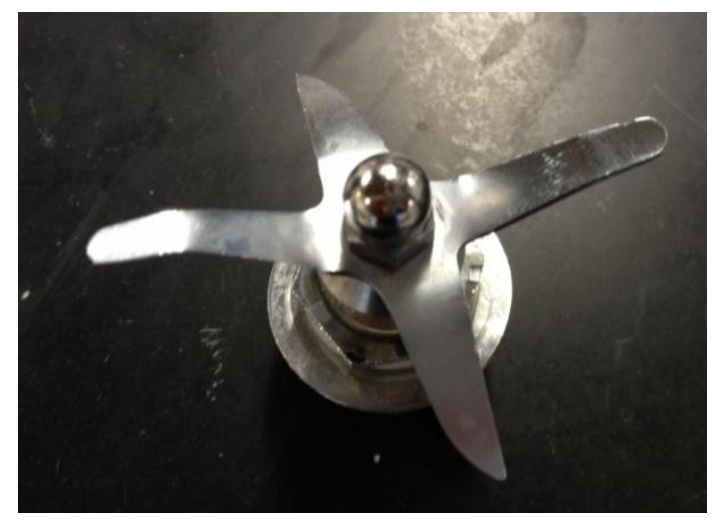

(a) Mixer blade assembly

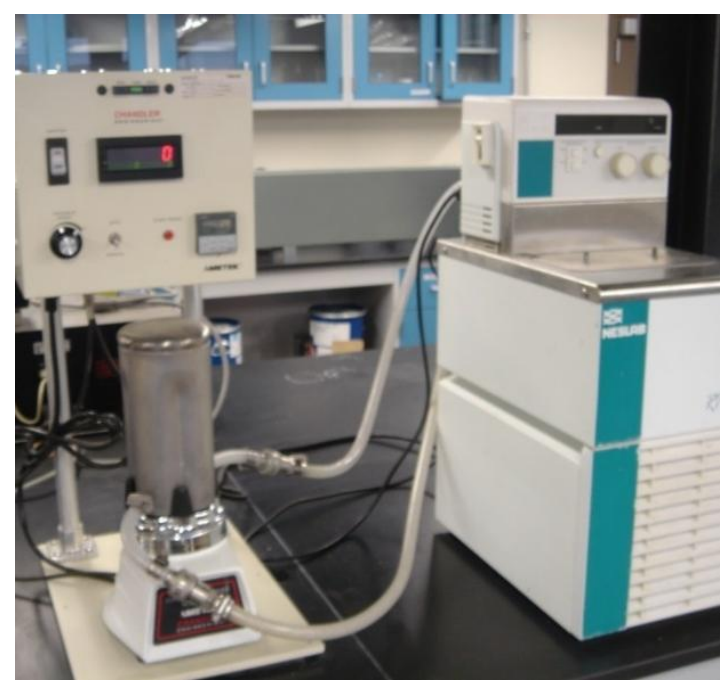

(b) High shear mixer connected to water circulation system

Figure 2. Chandler 3060 mixer system used to prepare cement pastes (a) blade set (b) mixer connected to water circulation system 


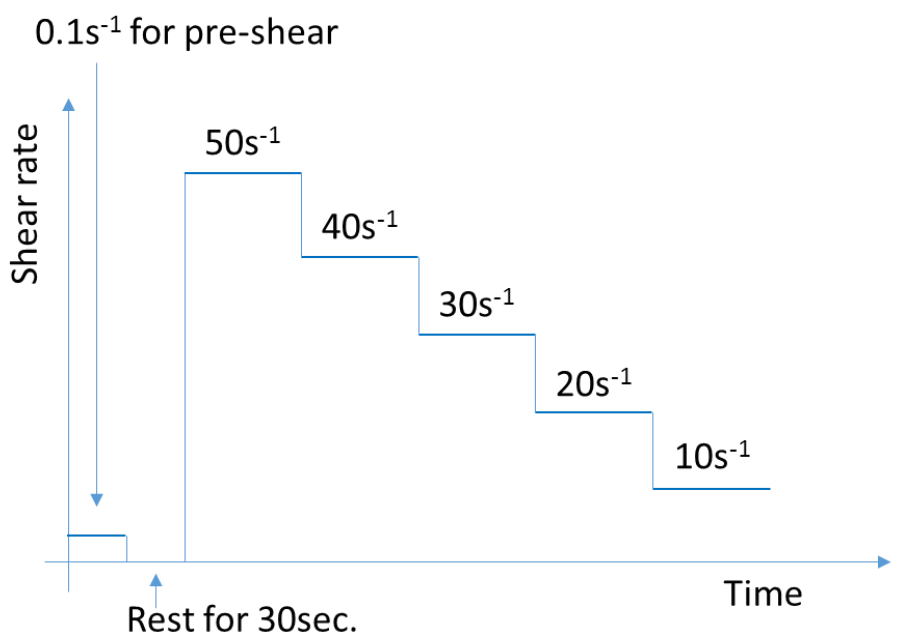

Figure 3. Applied shear rate protocol for flow curve measurement 34

35 


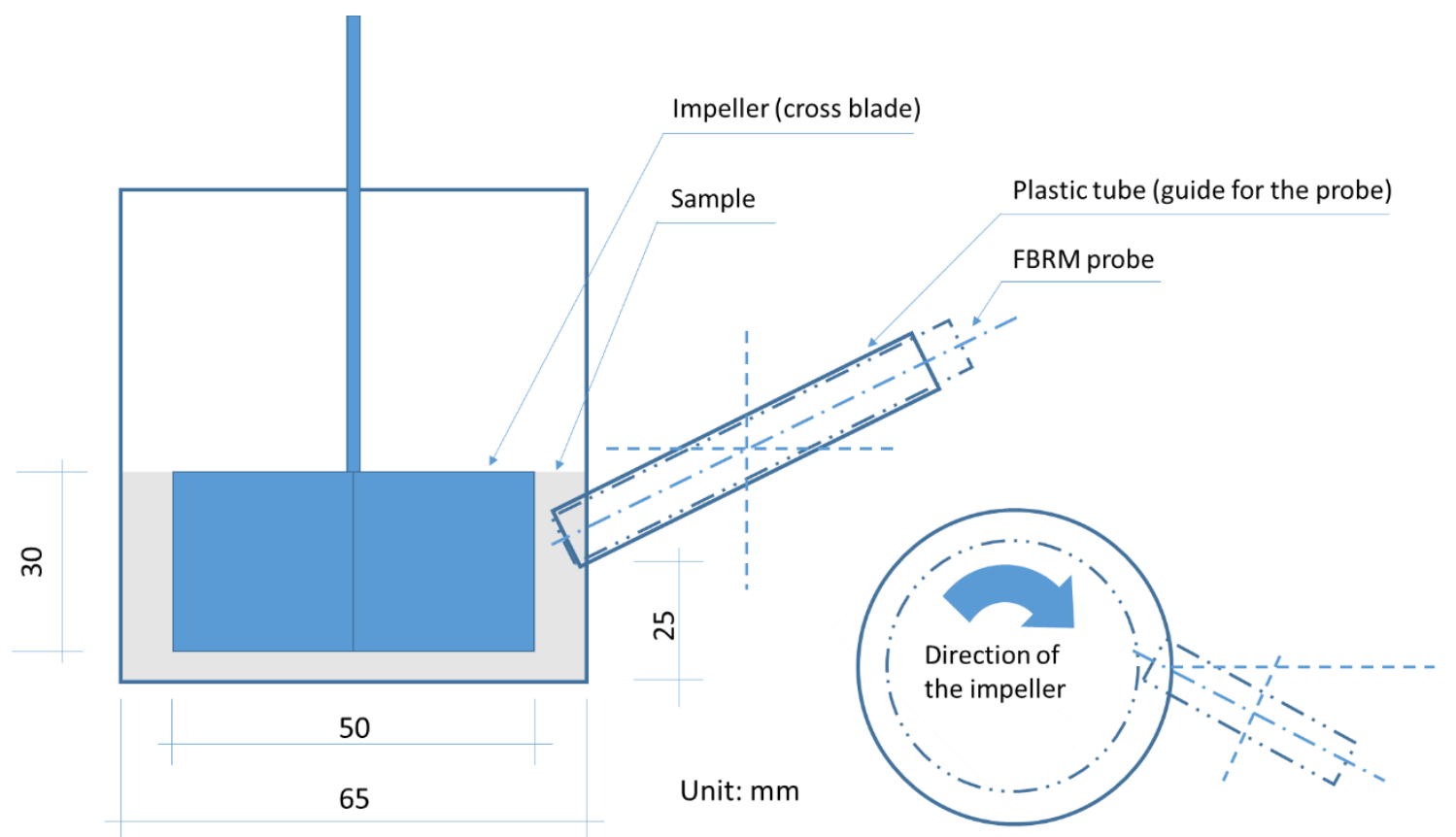

Figure 4. Schematic of FBRM test set up 


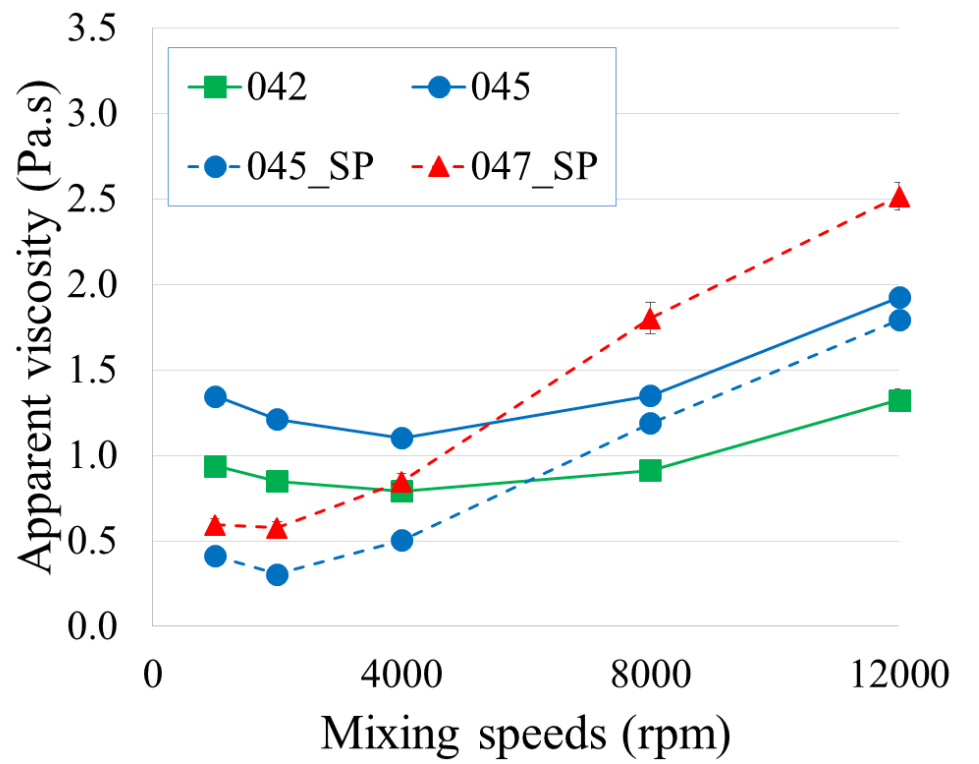

Figure 5. Influence of mixing speed and cement pastes on the apparent viscosity at $20 \mathrm{~s}^{-1}$ of shear rate (the data points were obtained by average of two representative flow curve tests) 


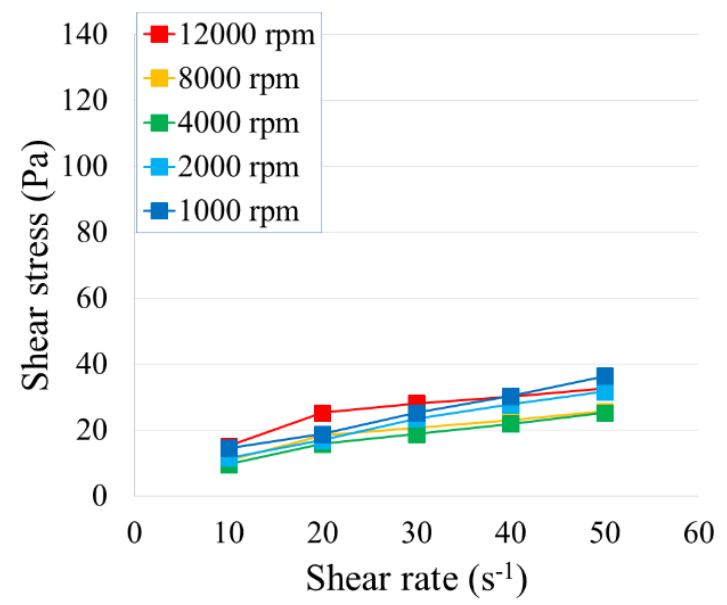

(a) 042

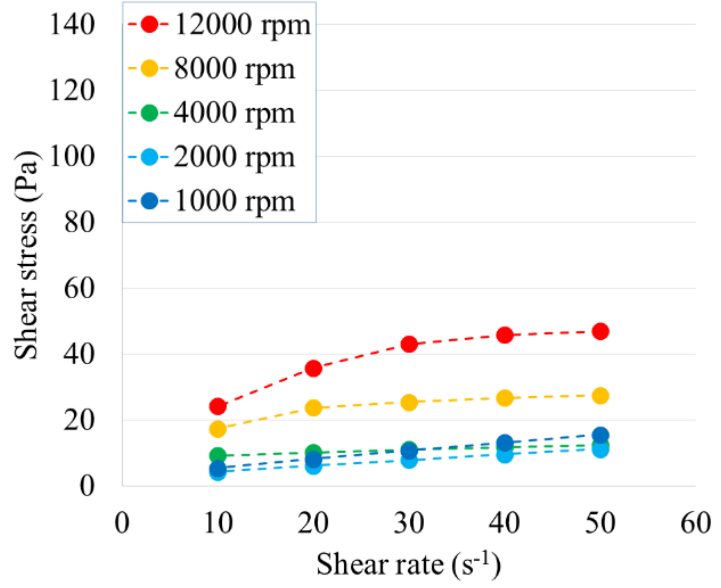

(c) 045_SP

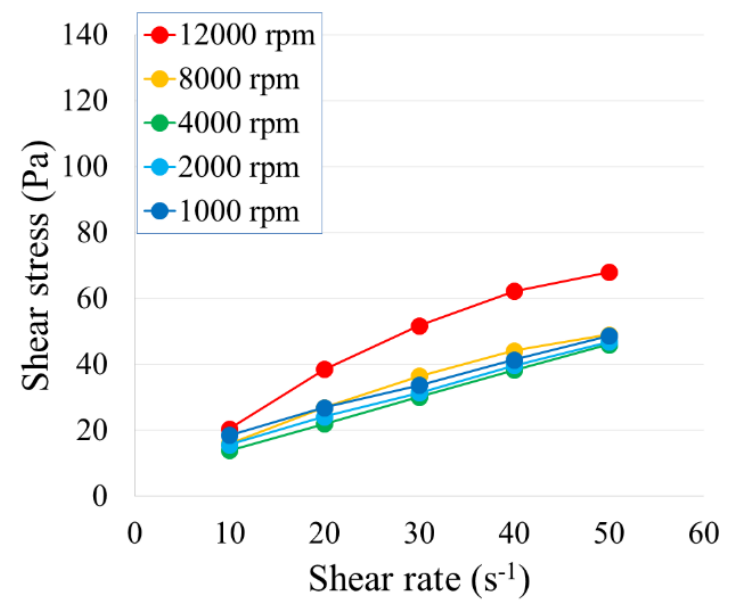

(b) 045

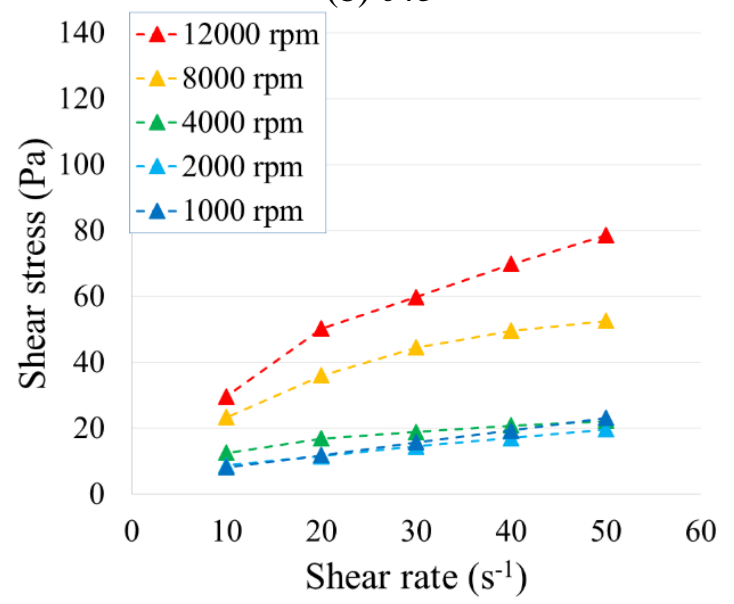

(d) 047_SP

40

Figure 6. Influence of mixing speed on flow curve behaviors depending on different cement pastes 


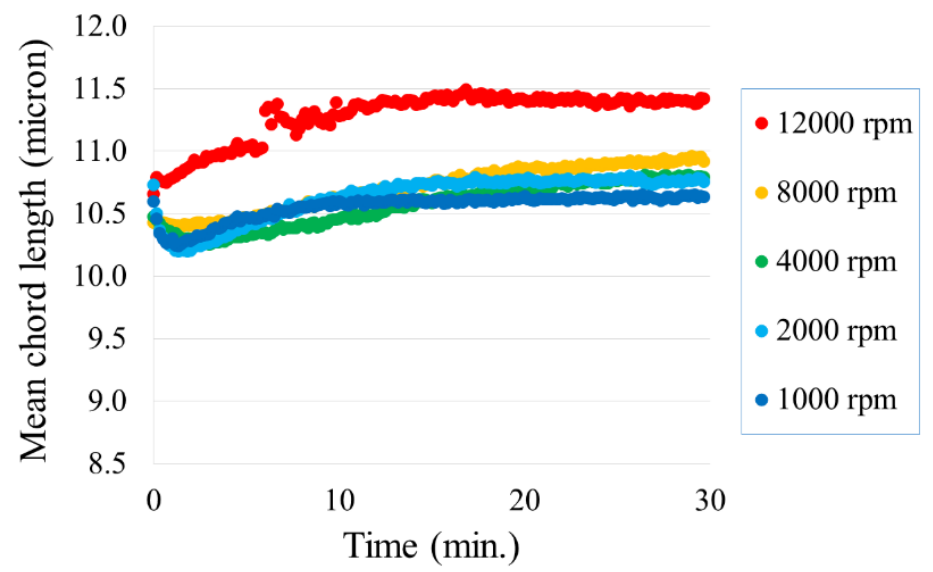

(a) FBRM observation speed:150 rpm

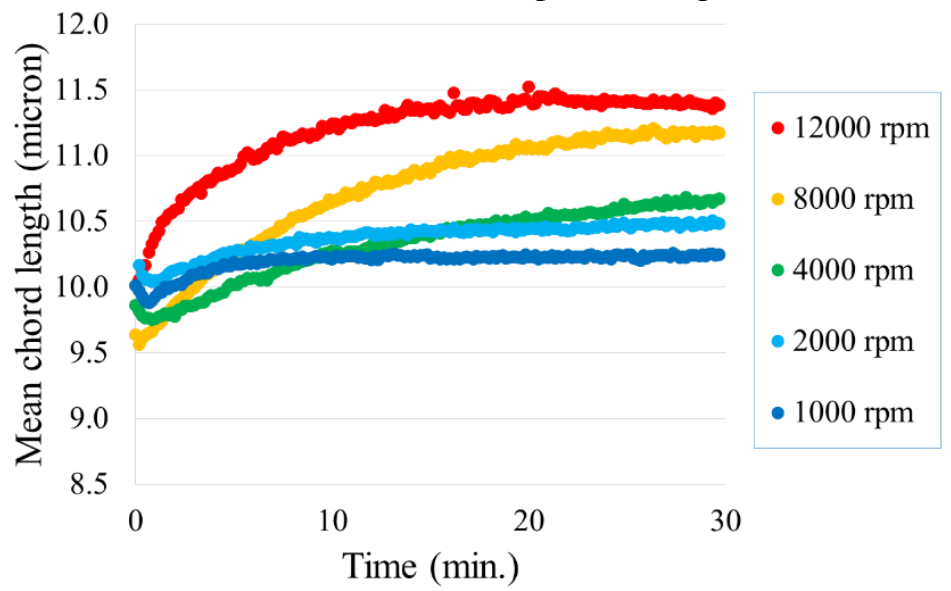

(b) FBRM observation speed:300 rpm

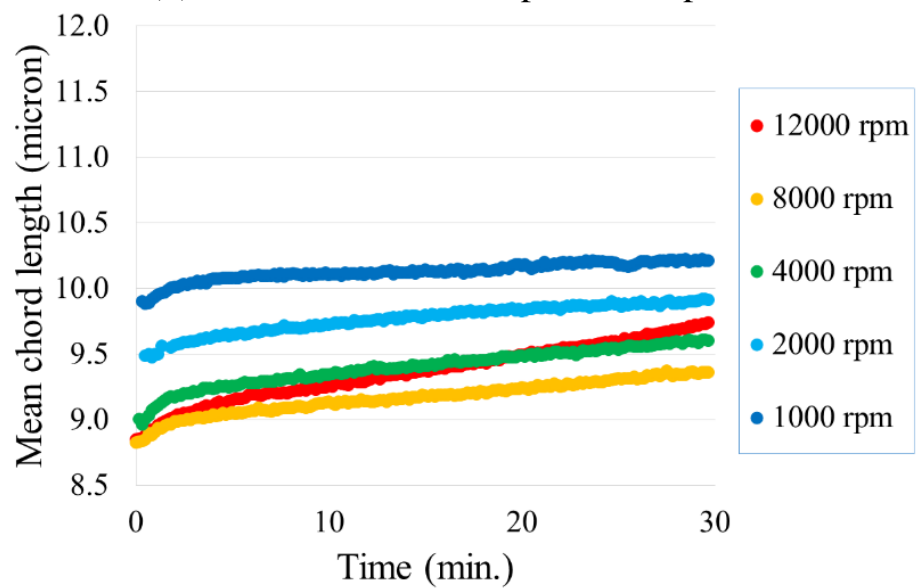

(c) FBRM observation speed:450 rpm

Figure 7. Influence of final mixing speeds on particle chord length under different agitation speeds for 042 paste 


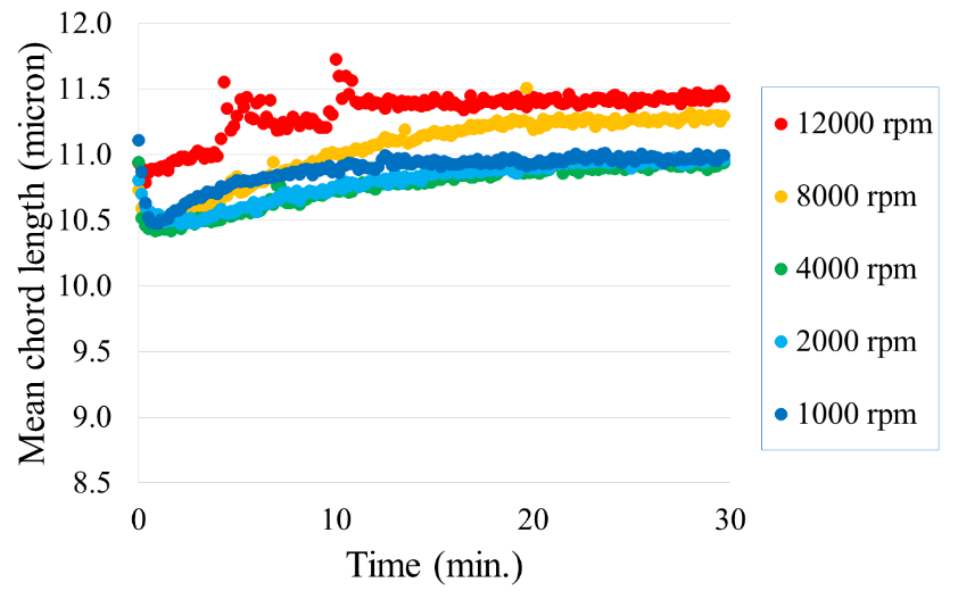

(a) $150 \mathrm{rpm}$

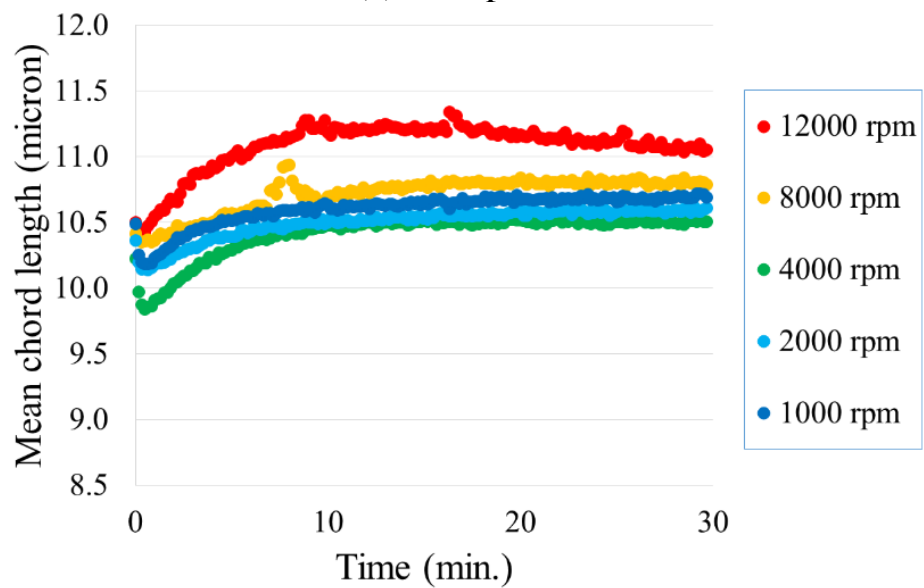

(b) $300 \mathrm{rpm}$

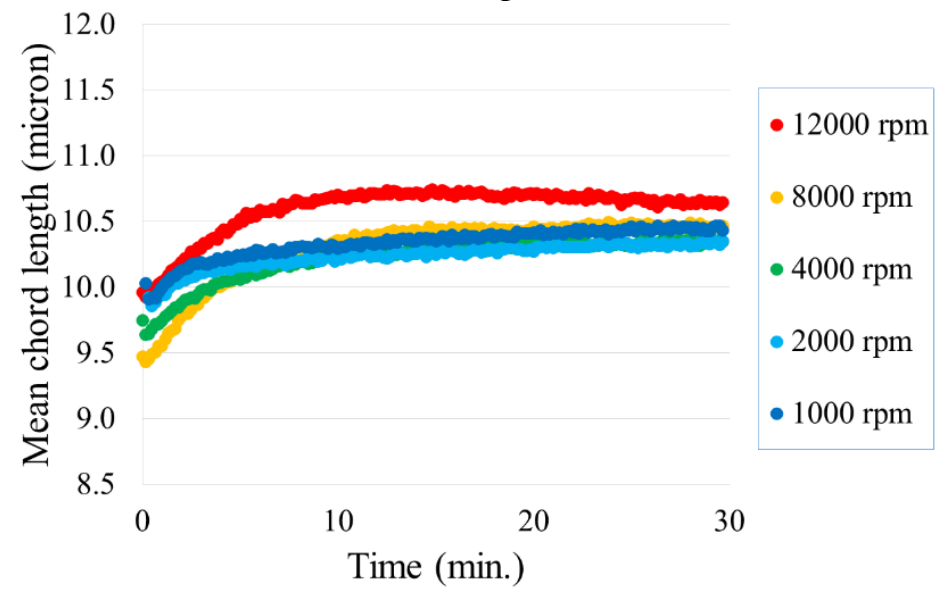

(c) $450 \mathrm{rpm}$

Figure 8. Influence of final mixing speeds on particle chord length under different agitation speeds for 045 paste 


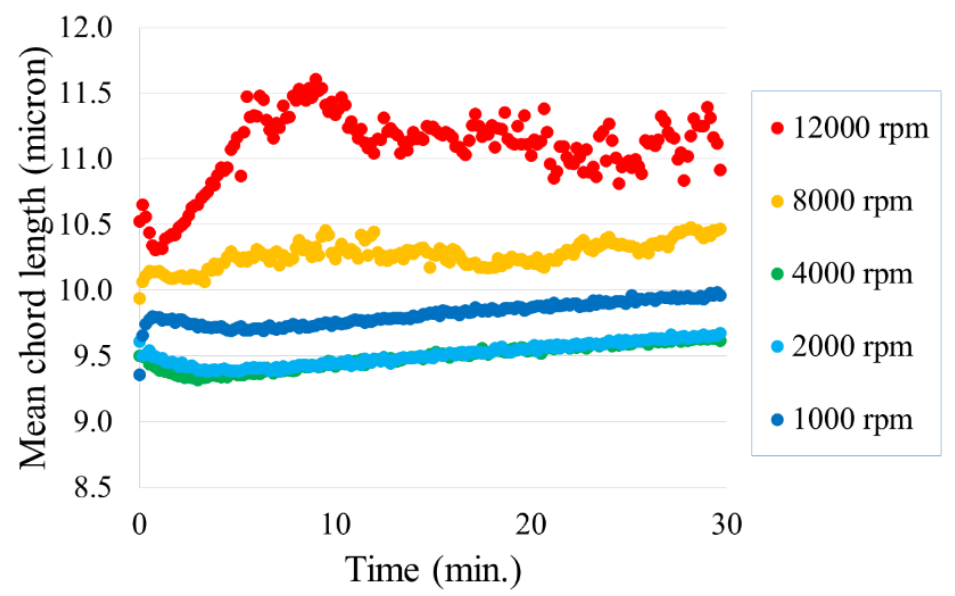

(a) $150 \mathrm{rpm}$

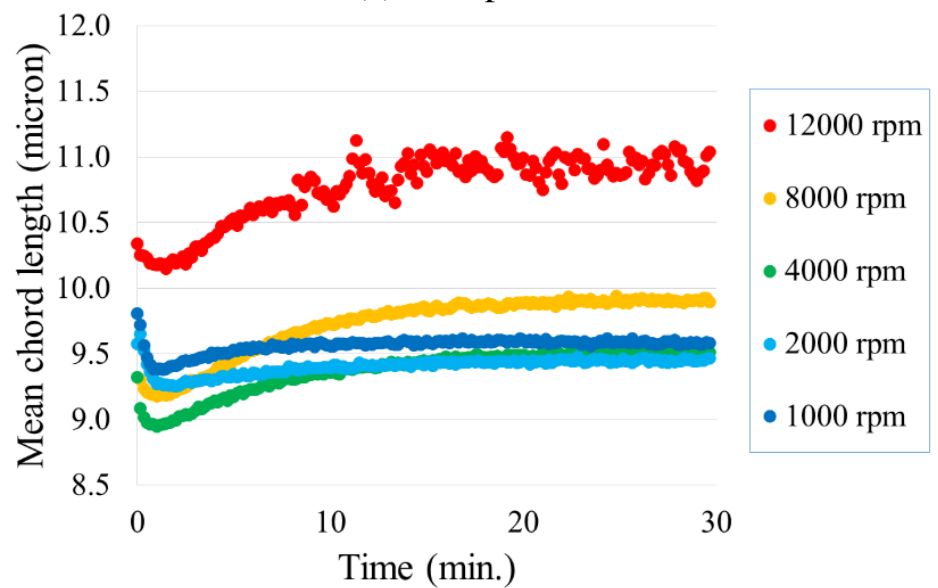

(b) $300 \mathrm{rpm}$

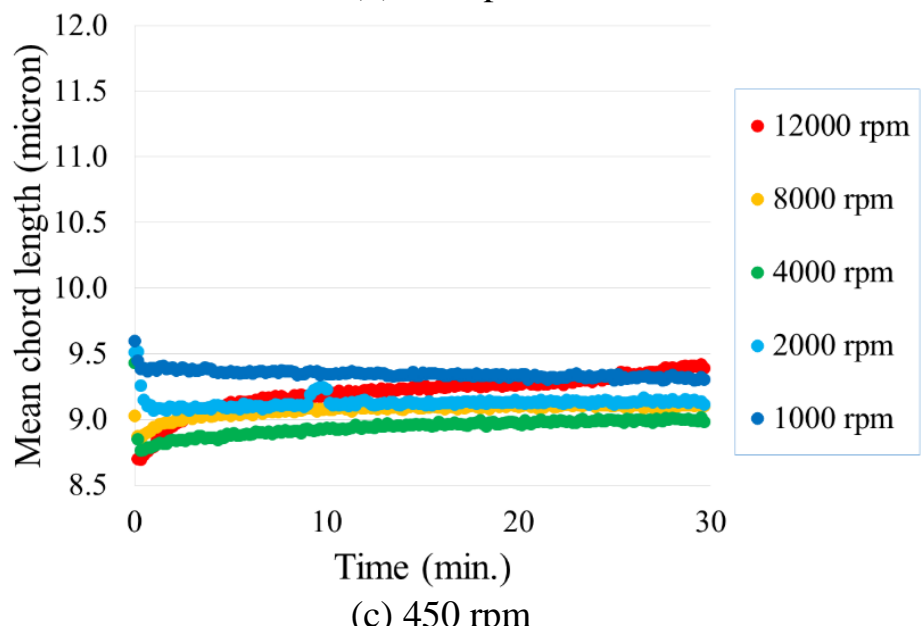

Figure 9. Influence of final mixing speeds on particle chord length under different agitation speeds for 045_SP paste 


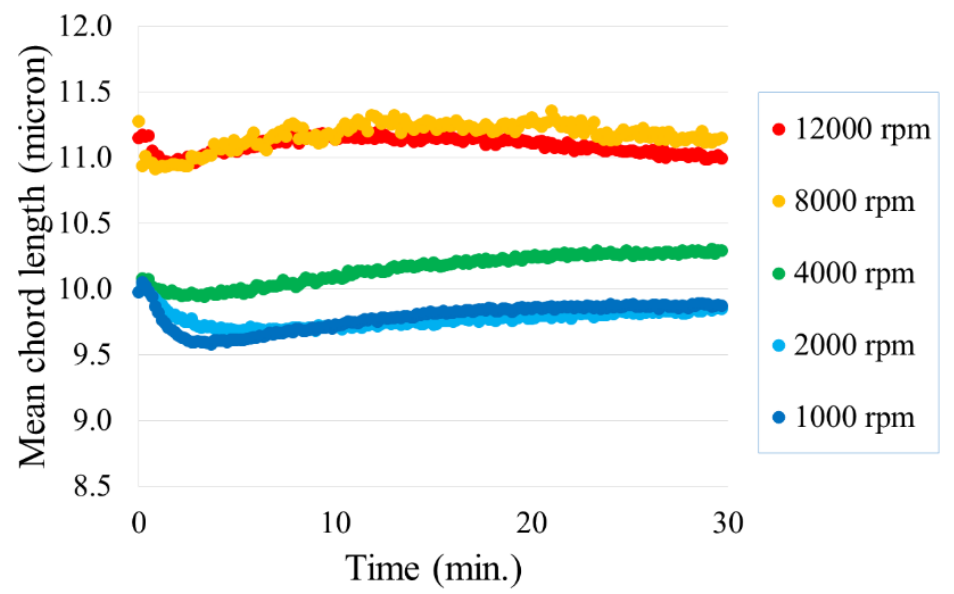

(a) $150 \mathrm{rpm}$

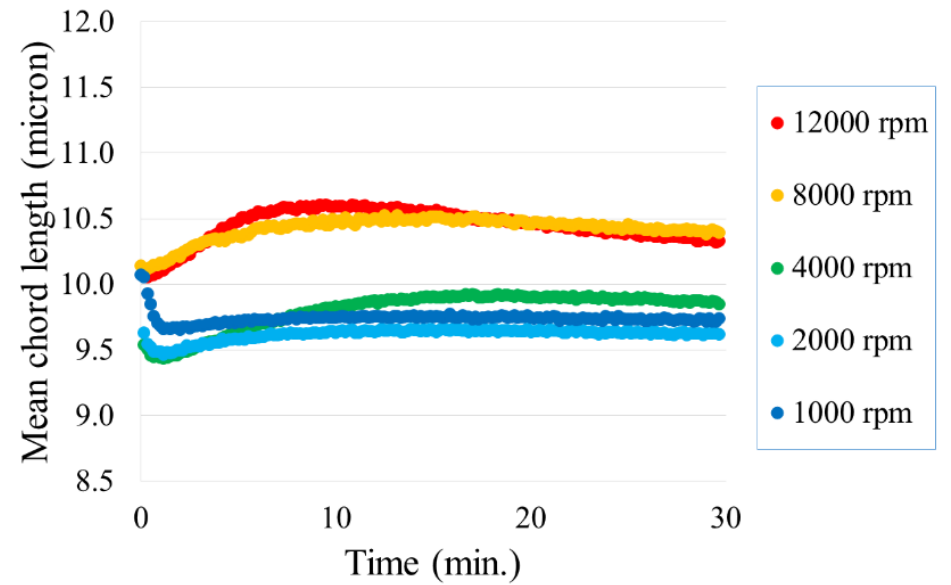

(b) $300 \mathrm{rpm}$

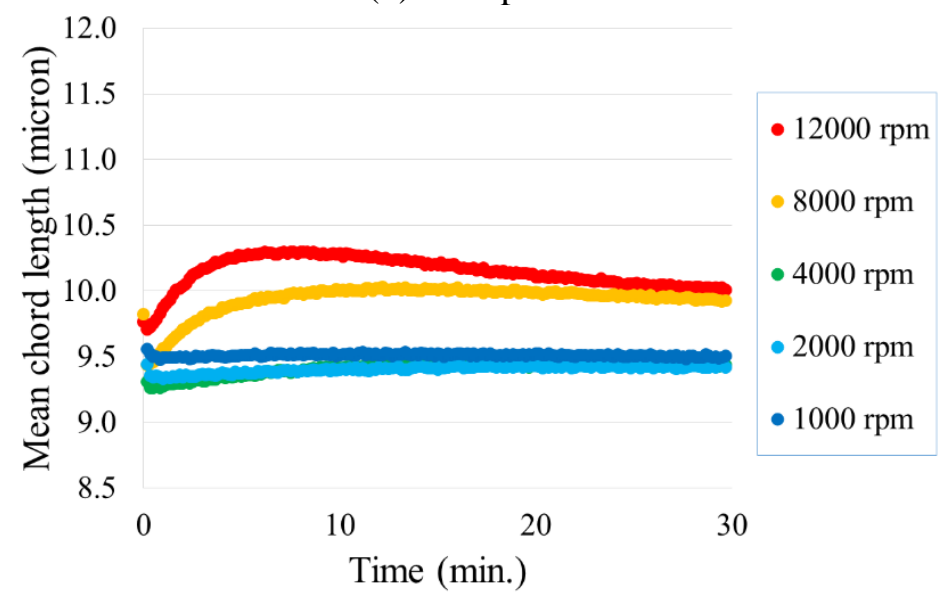

(c) $450 \mathrm{rpm}$

Figure 10. Influence of final mixing speeds on particle chord length under different agitation speeds for 047_SP paste 


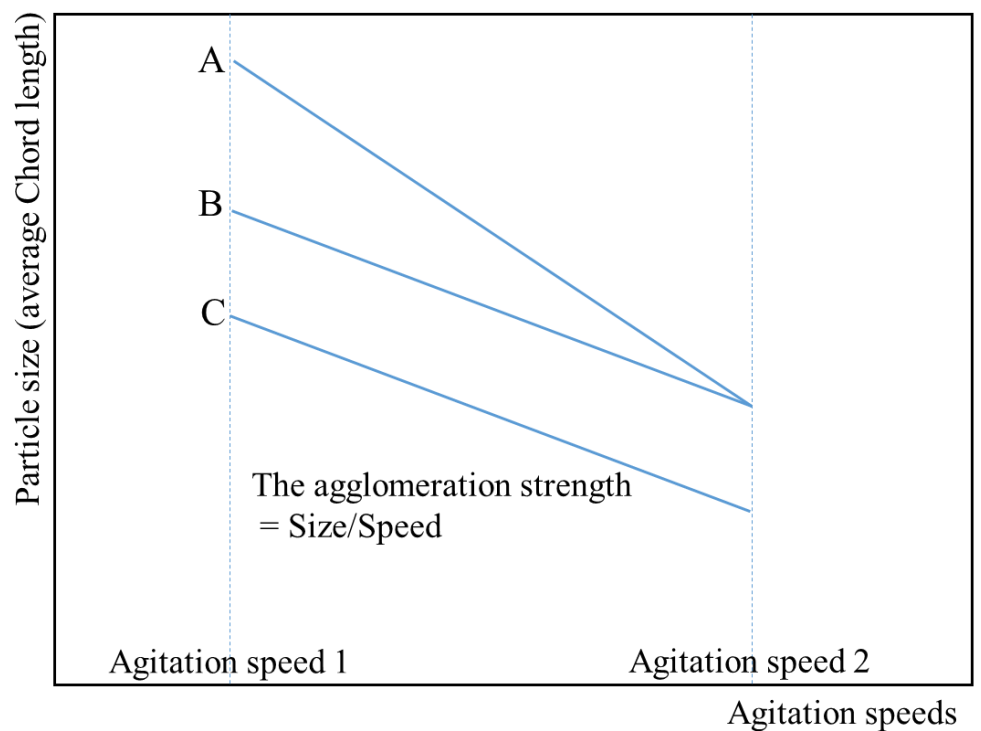

Figure 11. Schematic depicting how agglomeration strength of the cement agglomerates can be estimated using average particle chord length and agitation speeds from FBRM experiments 


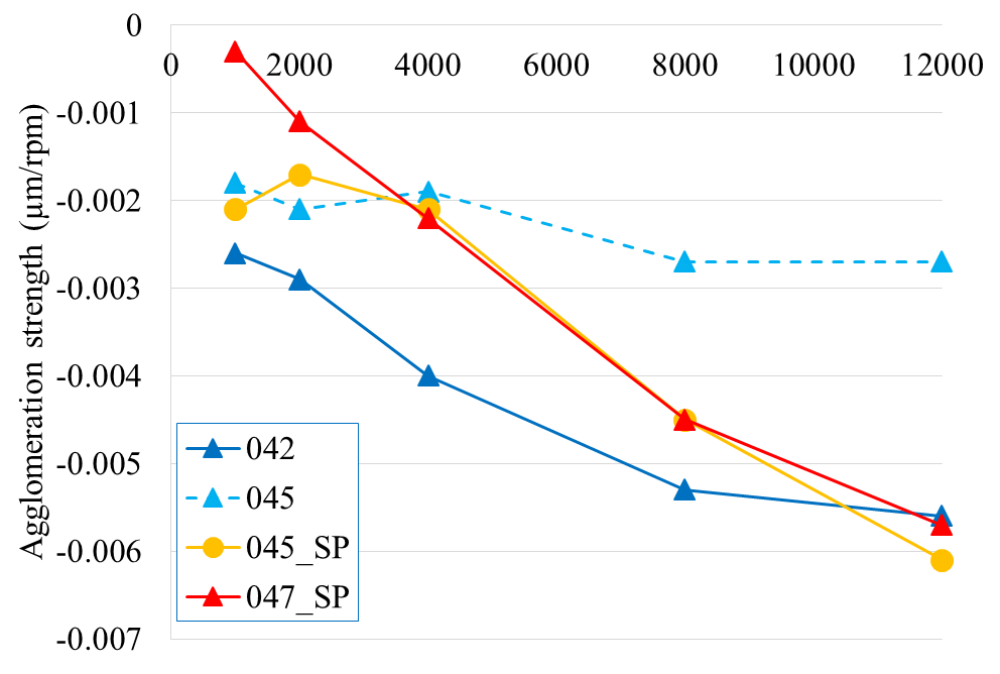

Mixing speed (rpm)

Figure 12. Estimated agglomeration strength in different pastes prepared at different final mixing speeds (Negative values indicates aggregate break-down whereas positive values indicate aggregate build-up.) 


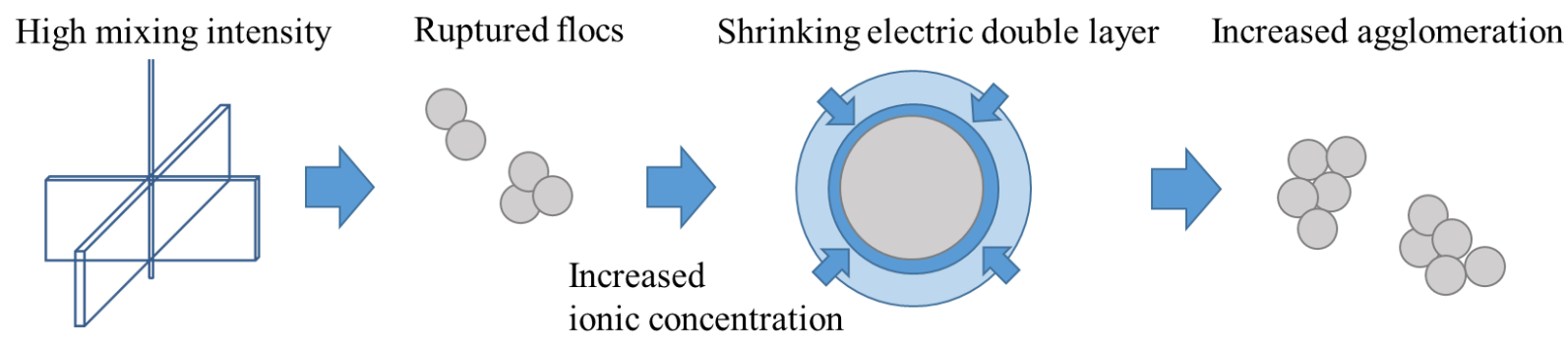
54

Figure 13. Schematic view of the hypothesis 


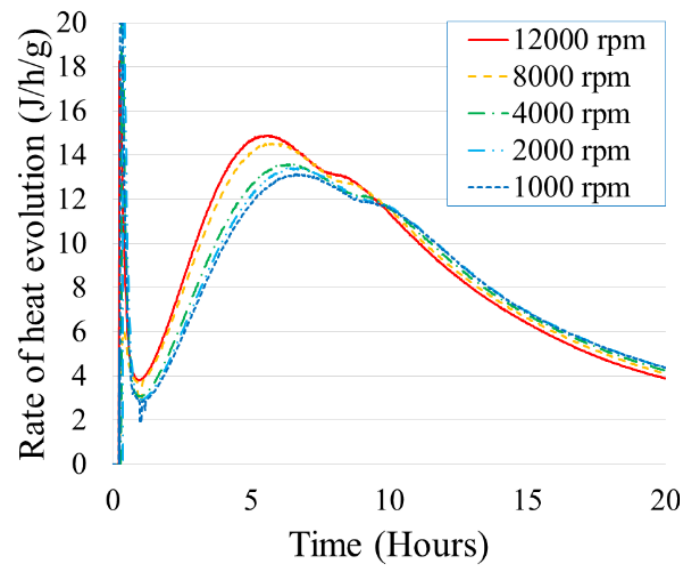

(a) 045

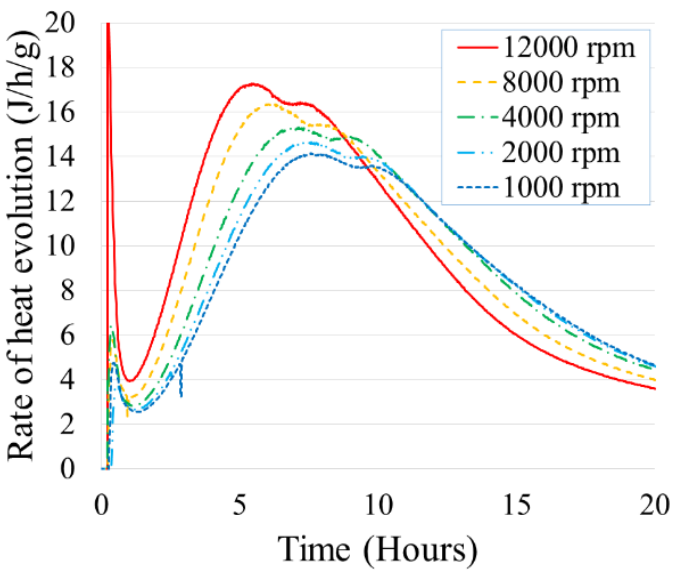

(b) 045_SP

Figure 14. Influence of the final mixing speeds on rate of heat evolutions of cement pastes 


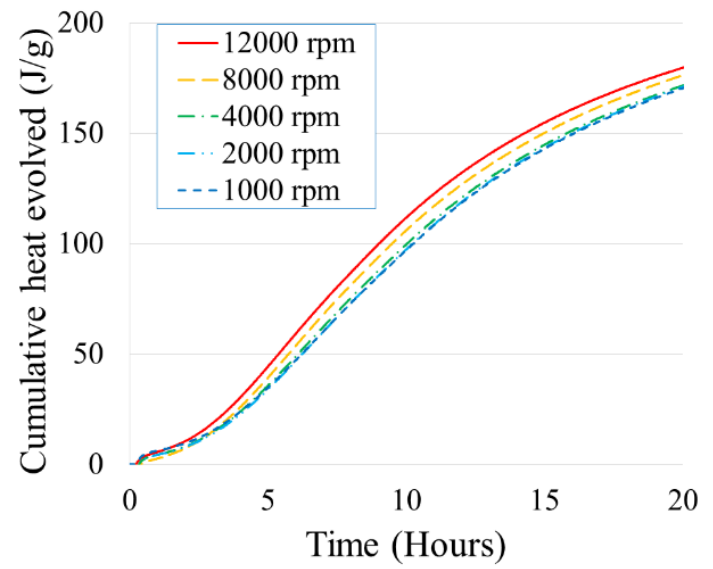

(a) 045

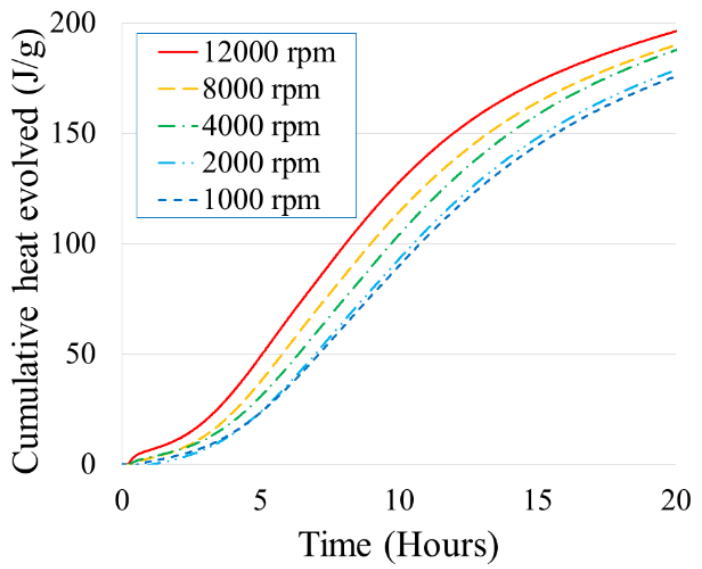

(b) 045_SP

Figure 15. Influence of the final mixing speeds on cumulative heat of hydration of cement pastes

58

59 


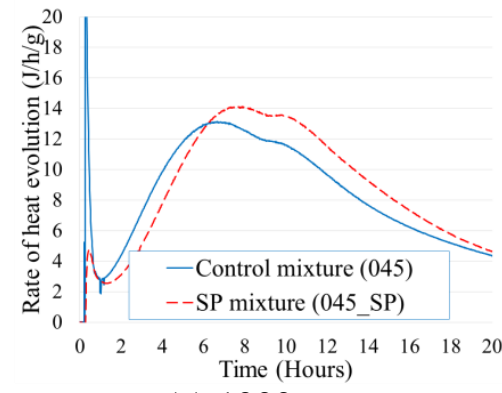

(a) $1000 \mathrm{rpm}$

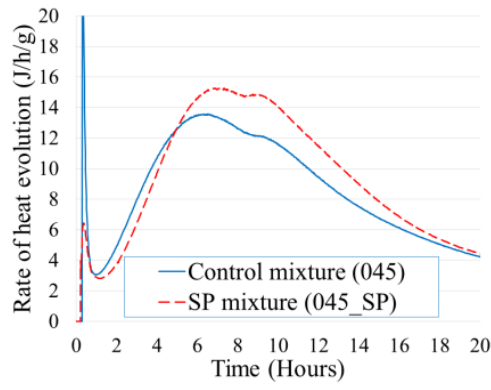

(b) $4000 \mathrm{rpm}$

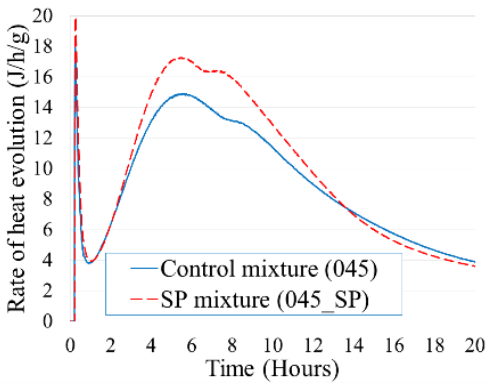

(c) $12000 \mathrm{rpm}$

60

Figure 16. Influence of mixing speeds on compensating the delaying effect of SP in cement pastes

61 


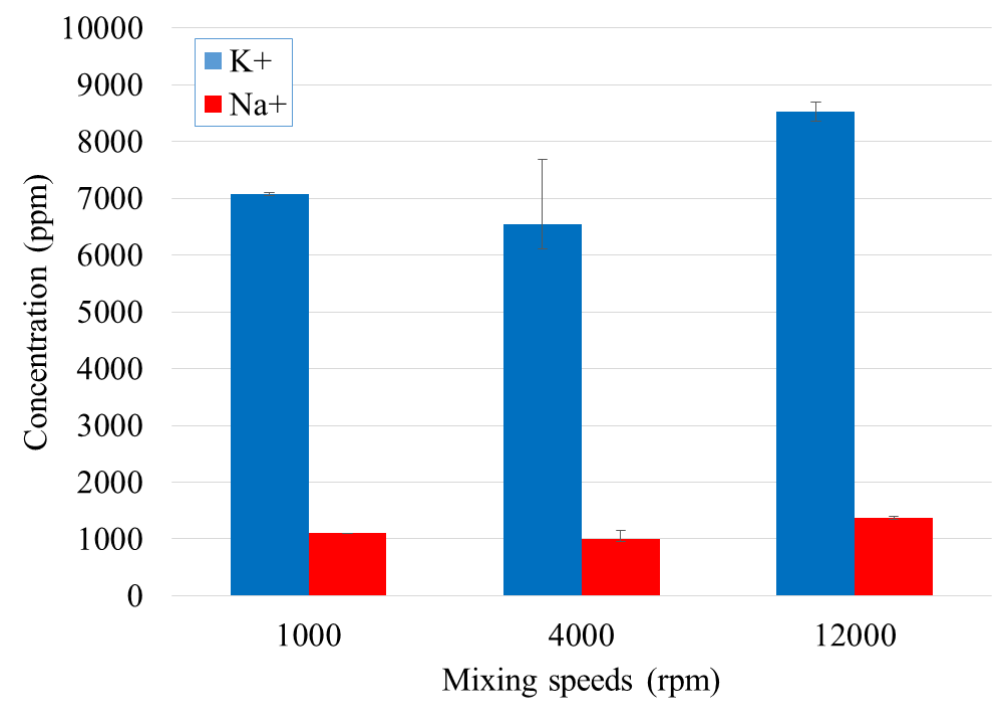

Figure 17. Influence of applied final mixing speeds on the concentration of alkalis from the fresh state 045 mixture

62

63 


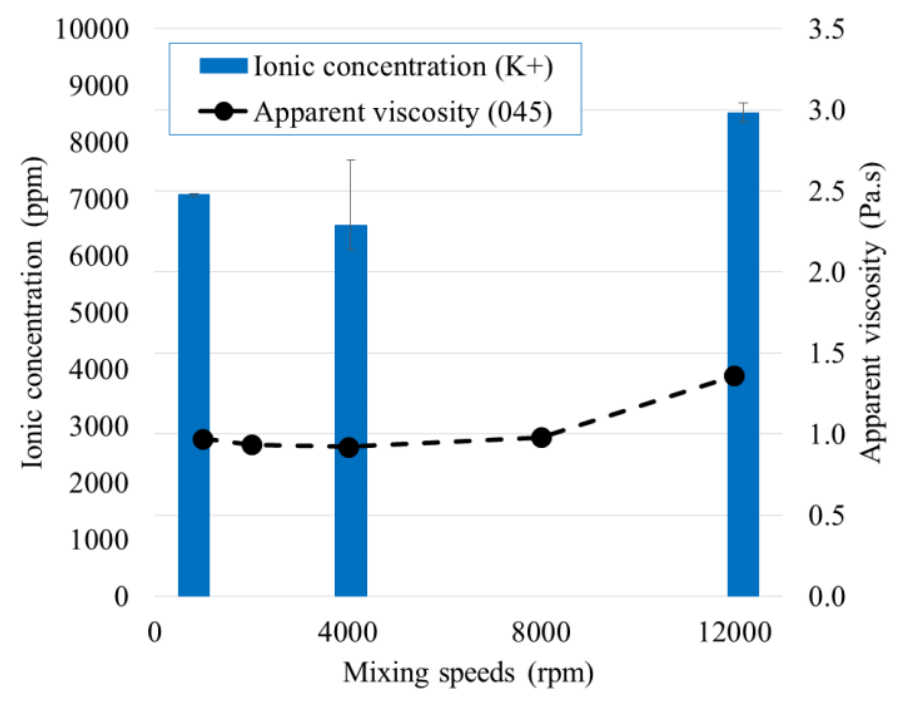

(a) $\mathrm{K}^{+}$

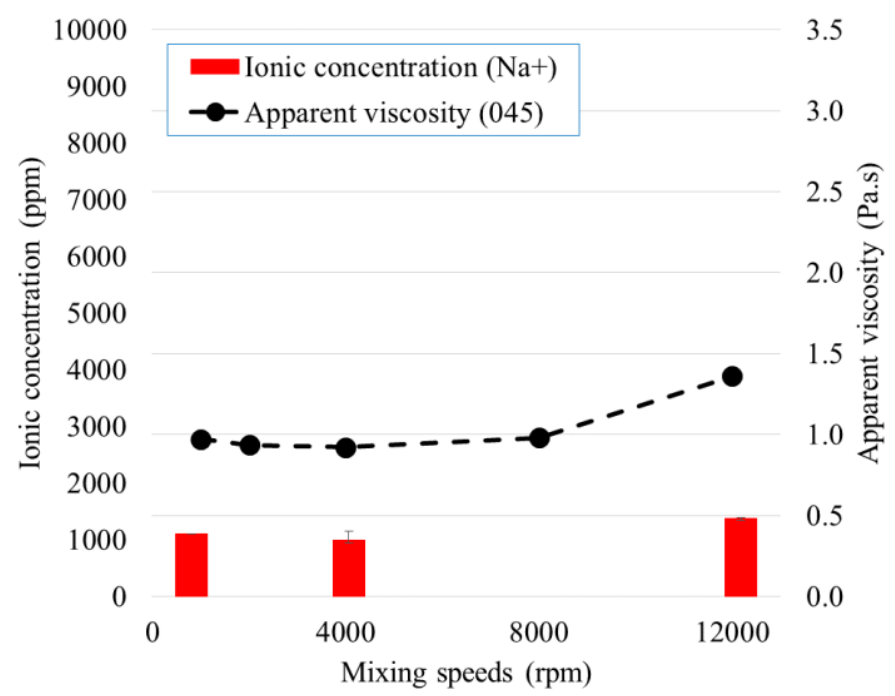

(b) $\mathrm{Na}^{+}$

Figure 18. Relationship between ionic concentrations of alkali ions and apparent viscosity of 045 mixture 